\title{
NF-kB-inducible miR-30b-5p aggravates joint pain and loss of articular cartilage via targeting SIRT1-FoxO3a-mediated NLRP3 inflammasome
}

\author{
Haiting $X u^{1}$, Jie Zhang ${ }^{2}$, Xiaoming $S^{2} i^{2}$, Xiaoyang $\mathrm{Li}^{1, \&}$, Chao Zheng ${ }^{3}$ \\ ${ }^{1}$ Department of Hand and Plastic Surgery, The Second Affiliated Hospital and Yuying Children's Hospital of \\ Wenzhou Medical University, Wenzhou 325027, Zhejiang, China \\ ${ }^{2}$ Department of Stomatology, Linyi People's Hospital, Linyi 276003, Shandong, China \\ ${ }^{3}$ Department of Reparative and Reconstructive Surgery, Linyi People's Hospital, Linyi 276003, Shandong, China
}

Correspondence to: Xiaoyang Li, Chao Zheng; email: lixioayang1166@163.com, doczheng77@163.com, https://orcid.org/0000-0002-4517-2290

Keywords: osteoarthritis, inflammation, miR-30b-5p, silent information regulator 2 homolog 1, NLRP3

Received: December 30, $2020 \quad$ Accepted: June 23, $2021 \quad$ Published: August 29, 2021

Copyright: (C) $2021 \mathrm{Xu}$ et al. This is an open access article distributed under the terms of the Creative Commons Attribution License (CC BY 3.0), which permits unrestricted use, distribution, and reproduction in any medium, provided the original author and source are credited.

\section{ABSTRACT}

MicroRNAs (miRNAs) contribute to osteoarthritis (OA) development. Nevertheless, the function and mechanism of miR-30b-5p in OA are unclear. In the present article, we gauged the miR-30b-5p level in OA patients and analyzed its correlation with $O A$ stages. Then, we conducted in-vivo and in-vitro gain-of-function assays to determine the function of miR-30b-5p, silent information regulator 2 homolog 1 (SIRT1) and Fox. Cell counting Kit-8 (CCK-8) assay, BrdU assay and flow cytometry were utilized to gauge cell viability and apoptosis of human chondrocyte (HC-A). The targeting association between miR-30b-5p and SIRT1 was validated through the dual-luciferase reporter assay and RNA immunoprecipitation (RIP) experiment. The results signified that miR-30b-5p was up-regulated in OA patients, OA rats and interleukin-1 $\beta$ (IL-1 $\beta$ )-induced chondrocytes. The higher miR-30b-5p expression brought about progressive stages of OA patients and enhanced levels of proinflammatory mediators in the synovial fluid. Functionally, overexpressing miR-30b-5p hampered cell viability, aggravated chondrocyte apoptosis and NLRP3 inflammasome activation induced by IL-1 $\beta$, while downregulating miR-30b-5p exerted the reverse effects. The in-vivo experiment exhibited that down-regulating miR$30 b-5 p$ improved joint pain and loss of articular cartilage in the rats with restrained inflammation and NLRP3 inflammasome activation. Mechanistically, miR-30b-5p targeted the 3'-non-translated region (3'UTR) of SIRT1, and miR-30b-5p was inducible with NF-KB phosphorylation enhancement. Overexpressing SIRT1 or inhibiting NF-KB relieved miR-30b-5p-induced apoptosis and NLRP3 inflammasome activation by promoting FoxO3a, while down-regulating SIRT1 or FoxO3a reversed miR-30b-5p-in-induced anti-inflammatory and apoptosissuppressive effects. Collectively, NF-kB-induced miR-30b-5p modulates chondrocyte apoptosis and OA progression by regulating the SIRT1-FoxO3a-mediated NLRP3 inflammasome.

\section{INTRODUCTION}

Osteoarthritis (OA) is a chronic arthropathy associated with articular degeneration, manifested as articular cartilage degeneration and bone hyperplasia, often found in the elderly $[1,2]$. OA is usually manifested as joint pain and poor movement, and subchondral ossification, trabecular fracture, and cystic changes are shown under X-ray examination [3]. In recent years, the $\mathrm{OA}$ incidence rate is on the rise due to population aging. Studies have stated that multiple inflammatory factors, such as TNF- $\alpha$, IL-6 and IL- $1 \beta$, contribute to OA development. Notably, the elevation of IL- $1 \beta$ facilitates the profiles of cyclooxygenase-2 (COX-2) and nitric oxide synthase (iNOS), thus expediting OA development [4]. Hence, it is crucial to inquiry into the 
specific mechanism of OA and reduce the inflammatory mediator release in OA treatment.

MicroRNAs (miRNAs) are noncoding single-stranded RNAs that are 18-25 nucleotides long. They regulate tumor progression, inflammation, angiogenesis, and so on. Meanwhile, miRNAs are implicated in OA evolvement. For instance, several studies have demonstrated that miRNA-34a induces synovium cell apoptosis in OA by down-regulating TGIF2 [5]. Additionally, $\mathrm{Li} \mathrm{F}$ et al. indicated that miRNA-103 enhances chondrocyte apoptosis and accelerates the OA process by reducing the SPHK1 activity and weakening the PI3K/AKT activation [6]. miR-30b-5p is a vital miRNA. Previous researches have claimed that NF- $\kappa B-$ mediated miR-30b regulation plays a key role in Ang IImediated cardiomyocytes targeting Bcl-2 [7]. Meanwhile, Yang $\mathrm{L}$ et al. have illustrated that cannabinoid receptor 1 (CB1) regulates the NLRP3 profile and the NLPR3 inflammasome activation in macrophages by regulating the miR-30b-5p axis, thereby easing liver inflammation [8]. Nevertheless, the action of miR-30b-5p in OA has not yet been discussed.

Silent information regulator 2 homolog 1 (SIRT1) is a nicotinamide adenine dinucleotide (NAD)-dependent deacetylase that modulates various pathways by deacetylating transcription factors. FoxO3a is the most significant member of the mammalian family of forkhead transcription factors [9, 10]. Studies have shown that miRNA-34a influences chondrocyte apoptosis and proliferation in OP pathogenesis by targeting SIRT1/p53 [11]. Besides, inhibiting miR301A eases LPS-induced chondrocyte injury by upregulating SIRT1 and activating the PI3K/AKT and NF$\kappa B$ pathways [12]. Bai $\mathrm{Y}$ et al. also showed that miR122 exerts an essential function in the extracellular matrix (ECM) degradation of OA chondrocytes by regulating the SIRT1 axis [13]. Nevertheless, whether miR-30b-5p contributes to OA by regulating SIRT1 and its transcription factor FoxO3a remains elusive.

Nod-like receptor family, pyrin domain containing 3 (NLRP3) inflammasome pertain to the NLR family, which is formed by NLRP3 scaffold, apoptosisassociated speck-like protein (ASC) and Caspase-1, and acts as an activating factor in the Caspase-1 pathway [14]. Increasing studies have demonstrated that NLRP3 inflammasomes produce pro-inflammatory cytokines and degrading enzymes like matrix metalloproteinase 3 (MMP-3), leading to cartilage degeneration and synovial inflammation [15]. Repressing NLRP3 inflammasome makes much sense in treating OA. For example, Dong $\mathrm{HC}$ et al. have stated that miR-223-3p directly targets NLRP3, and overexpressing miR-223$3 p$ restrains IL- $1 \beta$-induced chondrocyte apoptosis and inflammation, and overexpressing miR-223-3p restrains IL- $1 \beta$-induced chondrocyte apoptosis and inflammation [16]. Meanwhile, triptolide attenuates the malignant progression of OA by regulating miR-20b/NLRP3 [17]. However, whether miR-30b-5p enhances the role of NLRP3 inflammasome in the inflammatory progression of OA remains to be further explored.

Here, we discovered the miR-30b-5p profile in OA patients' joint fluid was significantly increased, and overexpressing miR-30b-5p aggravated chondrocyte apoptosis and inflammation in vitro. Therefore, both invitro and in-vivo experiments were implemented to probe the impact of miR-30b-5p on articular cartilage apoptosis in OA rats and its specific mechanism, thus providing some references for clinical research and treatment of OA from the molecular level.

\section{MATERIALS AND METHODS}

\section{Collection of patient tissues and specimens}

Cancerous tissues of 40 OA patients ( 17 females and 23 males, 55 65 years old) and matched normal samples from uninjured areas in 15 healthy donors were harvested from their knee joints by surgery in Linyi People's Hospital and preserved at $-80^{\circ} \mathrm{C}$. All patients signed informed consent forms, and all experiments were authorized by the Research Ethics Committee of Linyi People's Hospital and following the Declaration of Helsinki and institutional guidelines.

\section{Establishment of the OA model in SD rats}

All animal treatments were authorized by the Animal Care and Use Committee of Linyi People's Hospital. Forty ten-week-old male SD rats (Shanghai SLAC Laboratory Animal Co., Ltd.) were randomized into four groups: the sham group, the OA negative control $(\mathrm{OA}+\mathrm{NC}-\mathrm{in})$ group, the OA miR-30b-5p inhibitor group (OA+ miR-30b-5p-in), and the OA miR-30b-5p mimic group (OA+miR-30b-5p). The SD rat OA model was stimulated through surgical DMM [18, 19]. Briefly, the rat's right knee capsule in the $\mathrm{OA}+\mathrm{NC}$-in group, $\mathrm{OA}+$ miR-30b-5p-in group and $\mathrm{OA}+$ miR-30b-5p group was cut off from the tendon of the medial patella. The medial meniscus ligament was cut open with microsurgical scissors. In the sham group, the joint capsule was incised while the medial meniscus ligament was not damaged. The rats could eat and drink freely and were fed at $25 \pm 2^{\circ} \mathrm{C}$ with $50 \% \pm 15 \%$ humidity, with 12 hours of light/dark cycle. The rats in the $\mathrm{OA}+\mathrm{NC}$-in group and $\mathrm{OA}+\mathrm{miR}-30 \mathrm{~b}-5 \mathrm{p}$-in group were injected with 800 pmol of miR-30b-5p negative control or miR-30b-5p antagomir 6 hours before the surgery, and nine rats in each group were optionally selected and executed. Rat 
knees were collected for histopathological analysis for the following tests.

\section{Cell culture and transfection}

Human chondrocytes HC-A were bought from the Cell Center of the Chinese Academy of Sciences (Shanghai, China). Cells were grown in the RPMI1640 medium containing 10\% fetal bovine serum (FBS) and 1\% penicillin/streptomycin (Invitrogen, Carlsbad, CA, USA) at $37^{\circ} \mathrm{C}$ with $5 \% \mathrm{CO}_{2}$. RPMI1640 and FBS were obtained from Thermo Fisher Scientific (Waltham, MA, USA). In the logarithmic growth phase, $0.25 \%$ trypsin (Thermo Fisher HyClone, Logan, UT, USA) was adopted for trypsinization and sub-culture. As reported previously, IL-1 $\beta$ was applied to induce the OA chondrocyte model [20]. HC-A cell lines were treated with $5.0 \mathrm{ng} / \mathrm{mL}$ exogenous recombinant human IL-1 $\beta$ (R\&D Systems, Inc.) for 3, 6, 12, and 24 hours, respectively.

SIRT1 overexpression plasmid (SIRT1) and its corresponding negative control (vector), small inference RNA (si-RNA) SIRT1 (si-SIRT1), si-FoxO3a, miR30b-5p mimics, miR-30b-5p inhibitors (miR-30b-5pin), and the negative controls (si-NC and miR-NC) were constructed and synthesized by Guangzhou FulenGen Co., Ltd., China The NF-kB inhibitor BAY 11-7082 (Article No. HY-13453) was bought from MedChemExpress (Monmouth Junction, NJ, USA). HC-A cells in the logarithmic growth stage were inoculated in 24-well plates $\left(5 \times 10^{5} /\right.$ well). When the cell growth was stable, Lipofectamine 2000 (Invitrogen, Shanghai, China) was applied to transfect the above expression vectors into the cells. After 24 hours, the primary medium was substituted by a fresh and complete medium. The culture was continued for 48 hours at $37^{\circ} \mathrm{C}$ with $5 \%$ $\mathrm{CO}_{2}$, and the profiles of miR-30b-5p, SIRT1 and FoxO3a were compared by quantitative reverse transcription-polymerase chain reaction (RT-qPCR) or western blot (WB) to verify the transfection efficiency.

\section{RT-qPCR}

Total RNA was separated from tissues or cells with the TRIzol reagent (Invitrogen, Waltham, MA, USA). Nanodrop-spectrophotometer determined RNA concentration and purity. Then, the PrimeScRIPt-RT Kit (Promega, Madison, WI, USA) was utilized to transcribe $1 \mu \mathrm{g}$ of RNA into DNA. Next, SYBR®Premix-ExTaq $^{\text {TM }}$ (Takara, TX, USA) and ABI7300 were employed for RT-qPCR. The total volume of the PCR system was $30 \mu \mathrm{L}$ and $300 \mathrm{ng}$ of cDNA was included in each sample. The amplification was performed with an initial denaturation at $95^{\circ} \mathrm{C}$ for 10 minutes, with 45 cycles. All fluorescence data were quantified relatively. U6 was the endogenous control of miR-30b-5p, and GAPDH was that of SIRT1, IL-1 $\beta$, IL-6, TNF- $\alpha$, IL-18, and FoxO3a. RT-qPCR was conducted in triplicate. Guangzhou Ribo Biotechnology Co., Ltd., China synthesized the primers (Table 1).

\section{Western blot (WB)}

HC-A cells or the cartilage tissue of rats were harvested, rinsed with cold PBS three times, and added to 100 200 $\mu \mathrm{L}$ RIPA lysate (Beyotime Biotechnology, Shanghai, China). The cells underwent ultrasonic water-splitting, and the protein content was quantified by the Bradford method. The same amount of protein in each group was subjected to $10 \%$ SDS-PAGE and then transferred to PVDF membranes (Millipore, Bedford, MA, USA). Afterward, the primary antibodies (1:1000) of Bax (ab32503), cleaved-Caspase3 (ab2302), NLRP3 (ab214185), ASC (ab180799), cleaved Caspase-1 (ab74279), MMP3 (ab52915), MMP13 (ab39012), SIRT1 (ab189494), FoxO3a (ab109629), NF-kB p65 (ab207297), p-NF-kB p65 (ab239882), and GAPDH (ab181602) were added and incubated at $4^{\circ} \mathrm{C}$ overnight. After the membranes were cleaned twice with TBST, they were incubated at room temperature for 1 hour with fluorescein-labeled Goat anti Rabbit IgG (ab205718, 1:2500). The above antibodies were obtained from Abcam (Cambridge, UK). At last, the membranes were rinsed three times, exposed with enhanced chemiluminescence (ECL) chromogenic agent (Millipore, MA, Bedford, USA), and captured with a membrane scanner.

\section{Cell counting kit-8 (CCK8) method}

In the logarithmic growth stage, HC-A cells were trypsinized $\left(2 \times 10^{3}\right.$ cells $\left./ \mathrm{mL}\right)$ and inoculated in 96-well plates $(100 \mu \mathrm{L}$ suspension/well), and each group had three duplicated wells. The 96-well plates were then further incubated. After 24 hours, $10 \mu \mathrm{L} \mathrm{CCK} 8$ solution (Beyotime Biotechnology, Shanghai, China) was added to each well and incubated for 1 hour. After that, a microplate reader was utilized to review the optical density (OD value) of each well (450 nm wavelength) at 24, 48, 72, and 96 hours.

\section{Flow cytometry}

After different treatments, HC-A cells were trypsinized and harvested via centrifugation $(1500 \mathrm{rpm}, 3 \mathrm{~min})$. The apoptosis detection kit (Shanghai Zeye Biotechnology Co., Ltd., China, article number: ZY140626) was utilized to process the collected cells. After rinsing cells with PBS twice, we added $400 \mu \mathrm{L}$ pre-cooled PBS, 10 $\mu \mathrm{L}$ AnnexinV-FITC, and $5 \mu \mathrm{L}$ PI, respectively. The cells underwent incubation at $4^{\circ} \mathrm{C} 30$ min away from light, and then flow cytometry was employed for 
Table 1. Primer sequences of each gene.

\begin{tabular}{lc}
\hline Gene name & Primer sequence (5 $\rightarrow$ 3) \\
\hline miR-30b-5p & forward: CAGTGCAGGGTCCGAGGT \\
FOXO3A & reverse: AAGCGCCTTGTAAACATCCTACA \\
& forward: AGCCAGTCTATGCAAACCCT \\
reverse: CCAACCCATCAGCATCCATG & forward: TATGCTCGCCTTGCTGTAGA \\
SIRT1 & reverse: AACCTGTTCCAGCGTGTCTA \\
& forward: CAGGGGCCACCACGCTCTTC \\
TNF- $\alpha$ & reverse: CTTGGGGCAGGGGCTCTTGA \\
& forward: ATGAACTCCTTCTCCACAAGCGC \\
IL-6 & reverse: GAAGAGCCCTCAGGCTGGACTG \\
& forward: TCCCTTCATCTTTGAAGAAGA \\
IL-1 $\beta$ & reverse: GAGGCCCCAAGGCCACAGG \\
& forward: TTCAAGACCAGCCTGACCAA \\
IL-18 & reverse: GCTCACCACAACCTCTACCT \\
& forward: TGATCTTCATGGTCGACGGT \\
GAPDH & reverse: CCACGAGACCACCACCTACAACT \\
U6 & forward: CTCGCTTCGGCAGCACA \\
& reverse: AACGCTTCACGAATTTGCGT \\
\hline
\end{tabular}

apoptosis assessment. The apoptotic cell percentage was calculated by computer software.

\section{Safranin O-fast green staining}

The knee joints were put in $4 \%$ poly formaldehyde and immobilized for 24 hours. After the joints were conventionally dehydrated, transparentized and paraffin-embedded, they were sectioned $(5 \mu \mathrm{M})$. The Safranin O/Fast Green staining kit (ICH World, Woodstock, MD, USA) was applied for Safranin O-fast green staining following the manufacturer's guidelines. Quantification of Safranin O-positive cartilage area and thickness was made, and Image-Pro Plus 6.0 (Media Cybernetics, Bethesda, MD, USA) was employed for histomorphometric analysis.

\section{Haematoxylin-eosin (HE) staining and immunohistochemistry}

Cartilage blocks and subchondral bones were cut into $1.0 \mathrm{~cm} \times 1.0 \mathrm{~cm} \times 0.5 \mathrm{~cm}$ pieces. Then, the pieces were fixed with neutral formalin solution for three days, decalcified with $30 \%$ formic acid solution for 14 days, and dehydrated with gradient ethanol. After paraffin embedding, the pieces were sectioned $(5 \mu \mathrm{M})$. The cartilage specimens were dewaxed, hydrated, and dyed with Harris alum hematoxylin (Fuzhou Maixin Biotechnology, China) for $5 \mathrm{~min}$. After being cleaned in $0.5 \%$ hydrochloric acid alcohol for $10 \mathrm{~s}$ and dyed with eosin for $40 \mathrm{~s}$, the specimens were dehydrated, transparentized, fixed with neutral balsam, and reviewed under a microscope. The chondrocyte nucleus was blue and other tissues were pink. For detecting $p-N F-\kappa B$ expression in the cartilage, immunohistochemistry was carried out according to the previous study [21]. The primary antibody was the Anti-Rabbit NF-kB p65 (phospho S276) antibody (ab194726, Abcam) and the secondary antibody is Goat Anti-Rabbit IgG H\&L (HRP) (ab205718, Abcam). Finally, the histopathological changes were observed under a light microscope.

\section{Dual-luciferase reporter assay}

pGL3-SIRT1-wild Type (SIRT1-WT) and pGL3SIRT1-mutant (SIRT1-MUT) reporter vectors were constructed by integrating target fragments of wild-type and mutant SIRT1 into pGL3 vectors (Promega, Madison, WI, USA). SIRT1-WT or SIRT1-MUT was co-transfected with miR-30b-5p or negative control. After 48 hours, luciferase activity was measured. All tests were conducted in triplicate.

\section{RNA immunoprecipitation (RIP) analysis}

RIP analysis was implemented with the EZMagna RIP RNA-binding protein immunoprecipitation kit (Millipore, Bedford, MA, USA) per the manufacturer's guidelines. The transfected HC-A cells were cracked and immunoprecipitated with the anti-human argonaute 2 (Ago2) antibody (Abcam, Cambridge, UK) and control IgG (the input group). After 48 hours of incubation, the co-precipitated RNA was separated and measured using RT-qPCR. 


\section{Data analysis}

Data in this study were processed by SPSS22.0 statistical software (SPSS Inc., Chicago, IL, USA). Three repetitive wells were set in each group, and the tests were made at least three times. Measurement data with normal distribution were represented as mean \pm standard deviation $(\mathrm{x} \pm \mathrm{s})$. Two groups of data were compared by $t$ test, and a correlation test was implemented by Pearson correlation analysis. The statistics were significant when $P<0.05$.

\section{Ethics statement}

Our study was authorized by the Research Ethics Committee of Linyi People's Hospital.

\section{Data availability statement}

The data sets utilized and analyzed during the current study are available from the corresponding author on reasonable request.

\section{RESULTS}

miR-30b-5p was up-regulated in the joint tissues of $\mathrm{OA}$ patients and correlated with pro-inflammatory responses

First, we collected the joint tissues of 15 healthy donors and $40 \mathrm{OA}$ patients and gauged the contents of miR-30b$5 p$ and inflammatory cytokines (IL- $1 \beta$, IL- 6 , TNF- $\alpha$, and IL-18) in the joint tissues through RT-qPCR. The results confirmed that the miR-30b-5p profile was higher in OA patients' joint tissues than that in normal cartilage tissues $(P<0.05$, Figure $1 \mathrm{~A})$. The above results also applied to inflammatory cytokines $(P<0.05$, Figure $1 \mathrm{~B}-1 \mathrm{E})$. Meanwhile, the profiles of SIRT1 and FoxO3a were significantly down-regulated $(P<0.05$, Figure $1 \mathrm{~F}, 1 \mathrm{G})$. Pearson linear correlation analysis demonstrated that miR$30 \mathrm{~b}-5 \mathrm{p}$ was positively related to the levels of IL-1 $\beta$, IL-6, TNF- $\alpha$, and IL-18 in the joint tissues of OA patients $(P<0.05$, Figure $1 \mathrm{H}-1 \mathrm{~K})$. However, miR-30b-5p was negatively linked with the contents of SIRT1 and FoxO3a $(P<0.05$, Figure $1 \mathrm{~L}, 1 \mathrm{M})$. These findings signified that the miR-30b-5p profile was heightened in the joint tissues of OA patients and was positively related to the proinflammatory response.

\section{miR-30b-5p expression was facilitated in OA rat

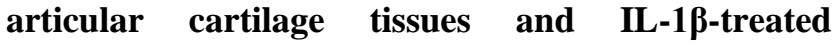 chondrocytes}

We constructed an OA model in SD rats through surgical DMM and tested the miR-30b-5p level in knee tissues of SD rats. RT-qPCR results illustrated that miR-30b-5p was distinctly up-regulated in the OA group (vs. the sham group) $(P<0.05$, Figure $2 \mathrm{~A})$. Furthermore, HC-A cells were intervened with $5 \mathrm{ng} / \mathrm{mL}$ $\mathrm{IL}-1 \beta$ for $3,6,12$, and 24 hours, respectively. As a result, the miR-30b-5p profile was elevated in HC-A cells after IL-1 $\beta$ administration time-dependently (vs. the control group) $(P<0.05$, Figure 2B). These findings confirmed that miR-30b-5p was highly expressed in OA cartilage tissues and chondrocytes.

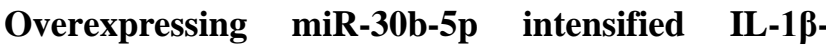 mediated chondrocyte apoptosis and inflammation}

To explore the effects of miR-30b-5p on IL-1 $\beta$-induced $\mathrm{OA}$, we transfected miR-30b-5p mimics and inhibitors into HC-A cells to construct miR-30b-5p overexpression and knockdown models. Meanwhile, the transfection validity was verified by RT-qPCR $(P<0.05$, Figure 3A). Cell viability was assessed by the CCK8 method, and the results manifested that HC-A cell viability was attenuated in the IL- $1 \beta$ group (vs. the control group). In addition, compared with the IL$1 \beta+$ miR-NC group, HC-A cell viability was further hampered after miR-30b-5p mimic transfection. Nevertheless, HC-A cell viability was elevated after transfection with miR-30b-5p inhibitors $(P<0.05$, Figure $3 \mathrm{~B})$. Flow cytometry results showed that HC-A cell apoptosis in the IL- $1 \beta$ group was evidently higher than that of the control group. In contrast, compared with the IL- $1 \beta+$ miR-NC group, HC-A cell apoptosis was further up-regulated by miR-30b-5p mimics. On the other hand, the miR-30b-5p inhibitor transfection had the reverse function $(P<0.05$, Figure $3 \mathrm{C})$. Furthermore, WB results found that the pro-apoptotic proteins Bax and CleavedCaspase 3 were up-regulated in the IL- $1 \beta$ group (vs. the control group). Besides, their expression was further enhanced after miR-30b-5p mimic transfection (vs. the IL- $1 \beta+$ miR-NC group). On the contrary, miR-30b-5p inhibitors exerted reversed effects $(P<0.05$, Figure 3D). Moreover, the expression of IL- $1 \beta$, TNF- $\alpha$, MMP 3 and MMP13 was compared by RT-qPCR and WB. It was found that the above-mentioned inflammatory cytokines were up-regulated in the IL- $1 \beta$ group (vs. the control group), and they were further up-regulated after miR$30 \mathrm{~b}-5 \mathrm{p}$ mimic transfection (vs. the IL- $1 \beta+$ miR-NC group). However, the results were completely reversed after the miR-30b-5p inhibitor transfection $(P<0.05$, Figure $3 \mathrm{E}, 3 \mathrm{~F})$. These results illustrated that miR-30b$5 \mathrm{p}$ up-regulation in human chondrocytes HC-A led to significant inflammation and apoptosis.

\section{The impact of abating miR-30b-5p on OA rats}

We transfected miR-30b-5p inhibitors to the knee joint cavity and gauged the miR-30b-5p profile by RT-qPCR 
to probe the effect of miR-30b-5p on $\mathrm{OA}$ in vivo. It turned out that miR-30b-5p expression was curbed after the miR-30b-5p inhibitor transfection into the OA model (vs. the miR-30b-5p negative control group) $(P<0.05$, Figure 4A). Meanwhile, HE and Safranin O staining were adopted to monitor morphological deviation of rat knee joint sections. As a result, compared with the sham group, the surface articular cartilage in the OA group was worn out, and the thickness of cartilage and the bony dermal plate was reduced. After supplementing miR-30b-5p inhibitors, the above situations were improved $(P<0.05$, Figure 4B). The contents of pro-inflammatory cytokines were further tested by RT-qPCR. The results confirmed that IL-1 $\beta$ and TNF- $\alpha$ expression in the OA group was heightened compared with that in the sham group, while they were down-regulated after the miR-30b-5p inhibitor transfection $(P<0.05$, Figure $4 \mathrm{C})$. Additionally, WB data testified that Bax, Cleaved-Caspase3, MMP3 and MMP13 in OA tissues were up-regulated (vs. the sham group). Nevertheless, the above proteins in the $\mathrm{OA}+\mathrm{miR}-30 \mathrm{~b}-5 \mathrm{p}$-in group were down-regulated in contrast with that in the $\mathrm{OA}+\mathrm{NC}$-in group $(P<0.05$, Figure 4D, 4E). IHC results showed that the NF- $\kappa B-$ positive cell number in the $\mathrm{OA}+\mathrm{miR}-\mathrm{NC}$-in group was heightened in contrast with the sham group, while the result was opposite after miR-30b-5p inhibition $(P<0.05$, Figure $4 \mathrm{~F})$. The profiles of $\mathrm{NF}-\kappa \mathrm{B}$ and SIRT1/FoxO3a were further gauged by WB. As a result, the phosphorylation of NF- $\kappa \mathrm{B}$ was evidently facilitated and the expression of SIRT1/FoxO3a was signally hampered in the OA+miR-NC-in group (vs. the sham group). However, the phosphorylation of NF- $\kappa \mathrm{B}$ was dramatically down-regulated and the SIRT1/FoxO3a profile was markedly facilitated after miR-30b-5p inhibition $(P<0.05$, Figure $4 \mathrm{G})$. These findings stated that attenuating miR-30b-5p restrained the inflammation in $\mathrm{OA}$ rats.
A

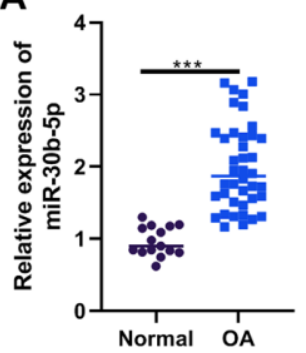

$\mathbf{F}$

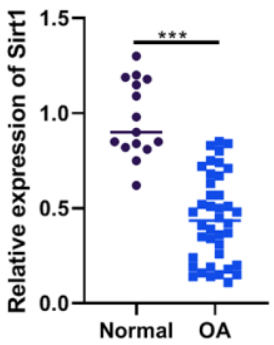

K

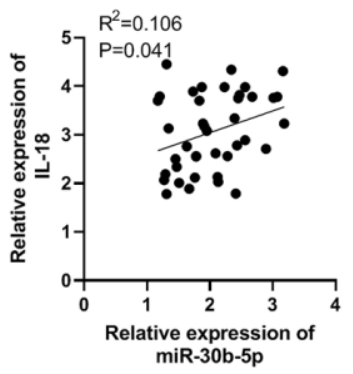

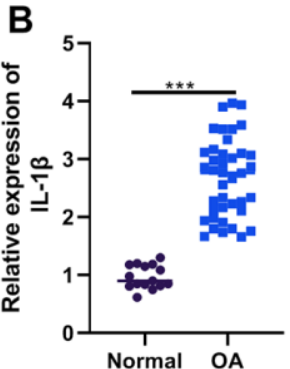

G
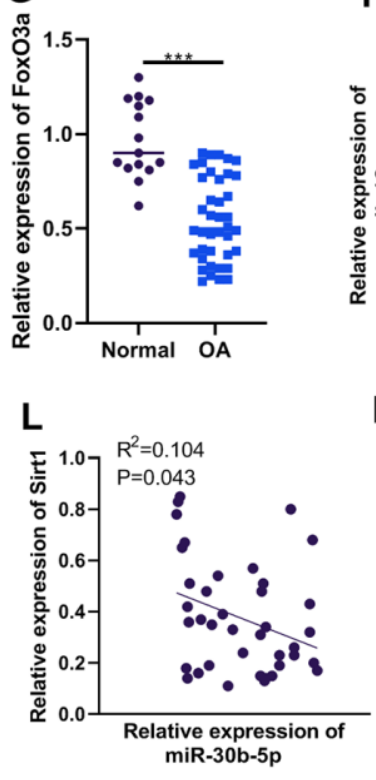

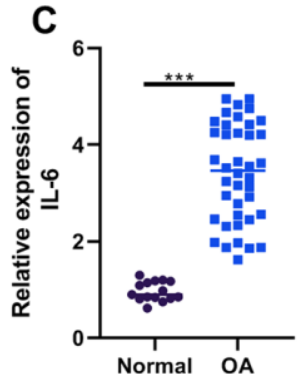

H
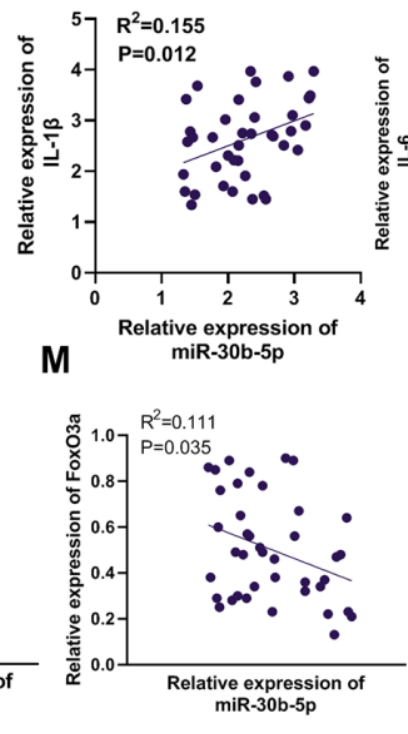

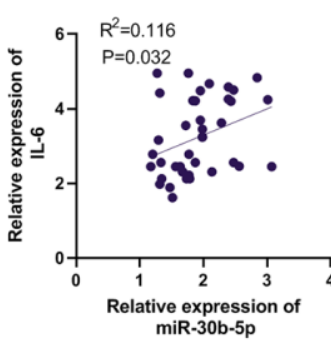

E
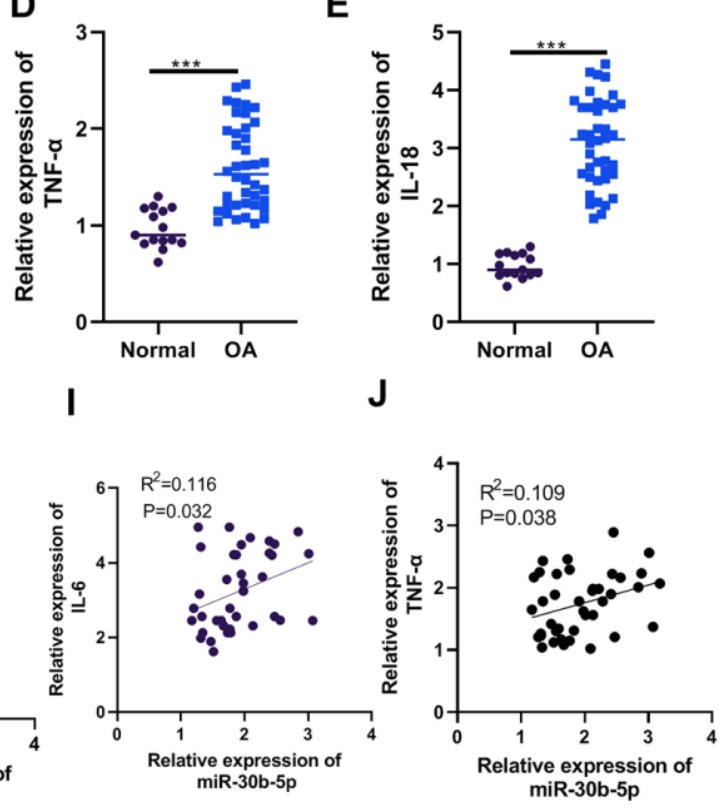

Figure 1. The miR-30b-5p profile was heightened in the joint tissues of OA patients and correlated with pro-inflammatory responses. The joint tissues of 15 non-OA patients and 40 OA patients were collected. (A-G) The levels of miR-30b-5p, IL-1 $\beta$, IL-6, TNF- $\alpha$, IL18, SIRT1 and FoxO3a in joint tissues were compared by RT-qPCR. (H-M) Pearson analysis determined the correlation between miR-30b-5p, inflammatory cytokines and SIRT1/FoxO3a in OA patients' joint tissues. ${ }^{* * *} P<0.001$ (vs. Normal group). 

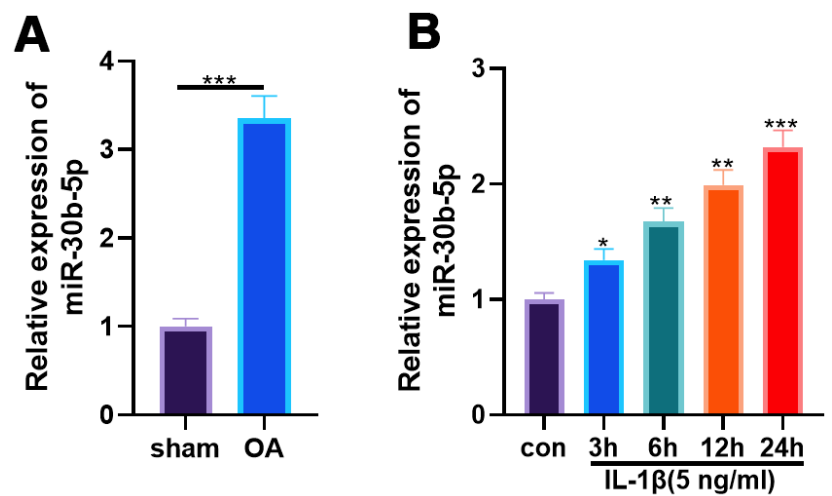

Figure 2. miR-30b-5p expression was heightened in OA rat articular cartilage tissues and IL-1 $\beta$-treated chondrocytes. DMM was induced to construct an SD rat model. (A) The miR-30b-5p profile in OA tissues was verified by RT-qPCR, N=5. IL-1 $(5 \mathrm{ng} / \mathrm{mL}) \mathrm{was}$ adopted to treat HC-A human chondrocytes. (B) RT-qPCR examined the miR-30b-5p profile in HC-A cells. ${ }^{*} P<0.05,{ }^{* *} P<0.01,{ }^{*} * * P<0.001$, vs. con group. N=3.

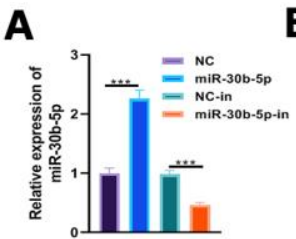

B

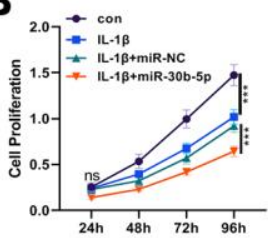

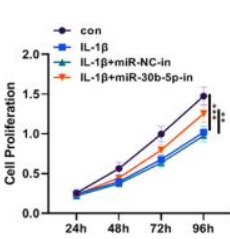

D

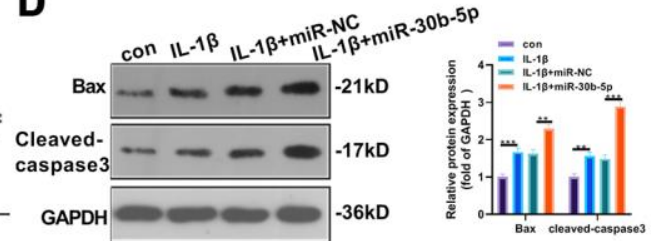

C
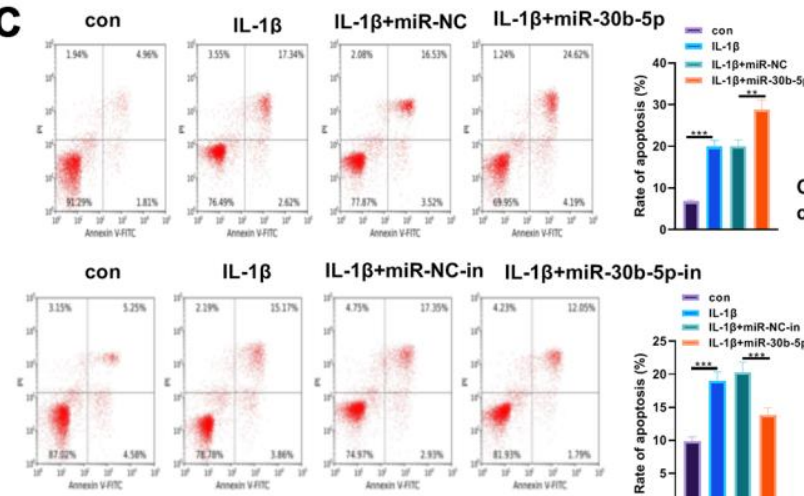

IL-1 $\beta+m i R-N C$-in IL-1 $\beta+m i R-30 b-5 p-i n$

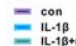

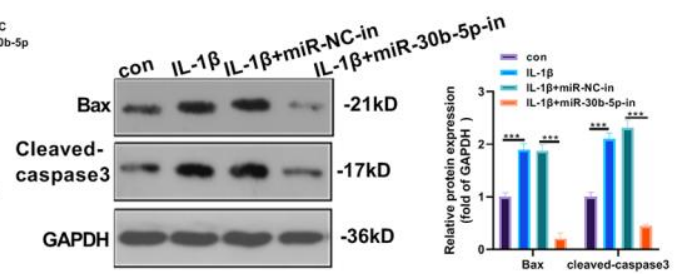

$\mathbf{E}$
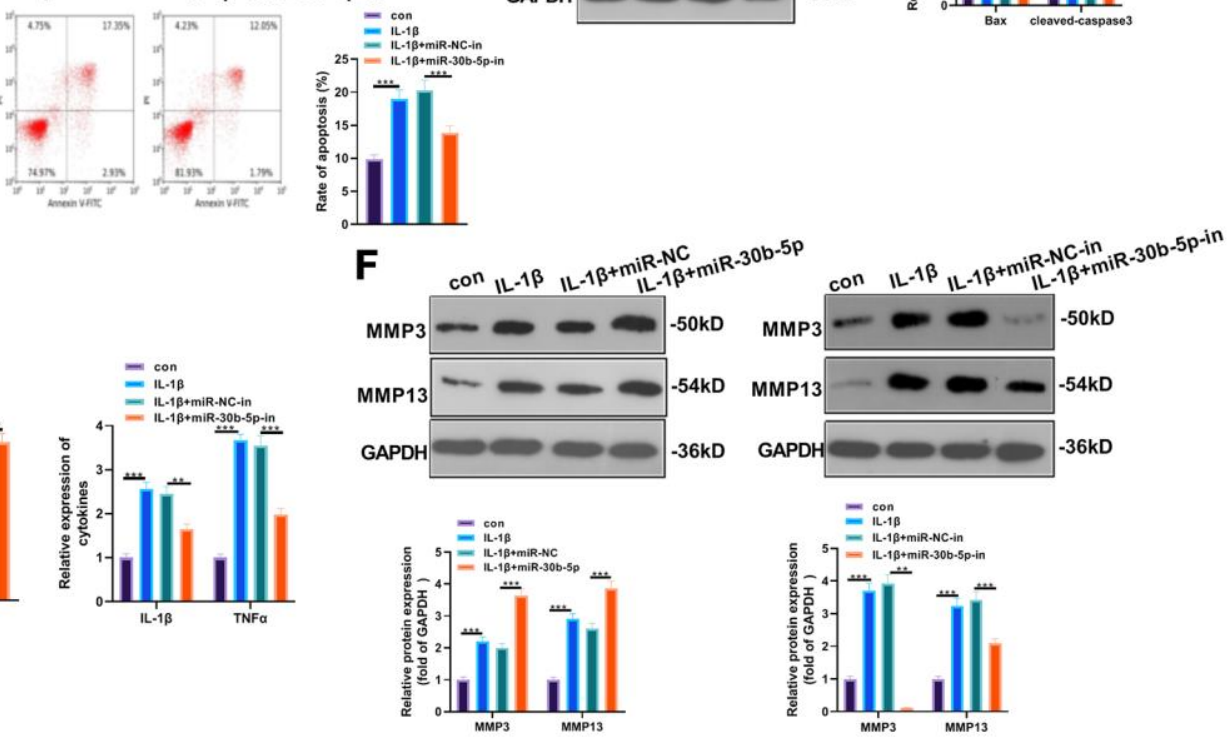

Figure 3. Overexpressing miR-30b-5p aggravated IL-1 $\beta$-mediated chondrocyte apoptosis and inflammation. miR-30b-5p mimics and inhibitors were transferred into HC-A cells, respectively. (A) RT-qPCR verified transfection validity. (B, C) CCK8 and flow cytometry tested cell viability and apoptosis, respectively. (D) Profiles of Bax and Cleaved-Caspase3 were examined by WB. (E, F) The expression of IL-1 $\beta$, TNF $\alpha$, MMP3, and MMP13 was monitored by RT-qPCR and WB. ${ }^{* *} P<0.01,{ }^{* * *} P<0.001 . \mathrm{N}=3$. 


\section{miR-30b-5p up-regulated NLRP3 in chondrocytes and joint tissues}

We adopted WB to examine the NLRP3 inflammasome expression in chondrocytes and joint tissues to probe the impact of miR-30b-5p on NLRP3. As a result, by contrast with the control group, NLRP3, ASC and cleaved-Caspase1 were significantly up-regulated in IL$1 \beta$-treated HC-A cells, and it was further up-regulated after miR-30b-5p mimics were added. On the contrary, it was down-regulated after miR-30b-5p inhibitors were supplemented $(P<0.05$, Figure 5A, 5B). Similarly, by contrast with the sham group, NLRP3, ASC and cleaved-Caspase1 were up-regulated in the OA group, while they were signally down-regulated after the supplementation of miR-30b-5p inhibitors into the knee cavity $(P<0.05$, Figure $5 \mathrm{C})$. These findings revealed that
miR-30b-5p up-regulated NLRP3 inflammasomes in the OA model in vivo and in vitro.

\section{SIRT1 targeted miR-30b-5p}

The common targets of SIRT1 were predicted via PITA, miRmap, microT and miRanda, and the shared miRNAs were validated by the Venn diagram. miR-30b-5p was discovered to be one of them (Figure 6A). Through the ENCORI database (http://starbase.sysu.edu.cn/), we discovered a binding site between miR-30b-5p and SIRT1 (Figure 6B). Furthermore, the dual-luciferase reporter assay results indicated that miR-30b-5p mimics hampered the luciferase activity of cells transfected with SIRT1-WT vectors but had little impact on that of SIRT1-MUT ( $P<0.05$, Figure 6C). Additionally, RIP results illustrated that the miR-30b-5p mimic
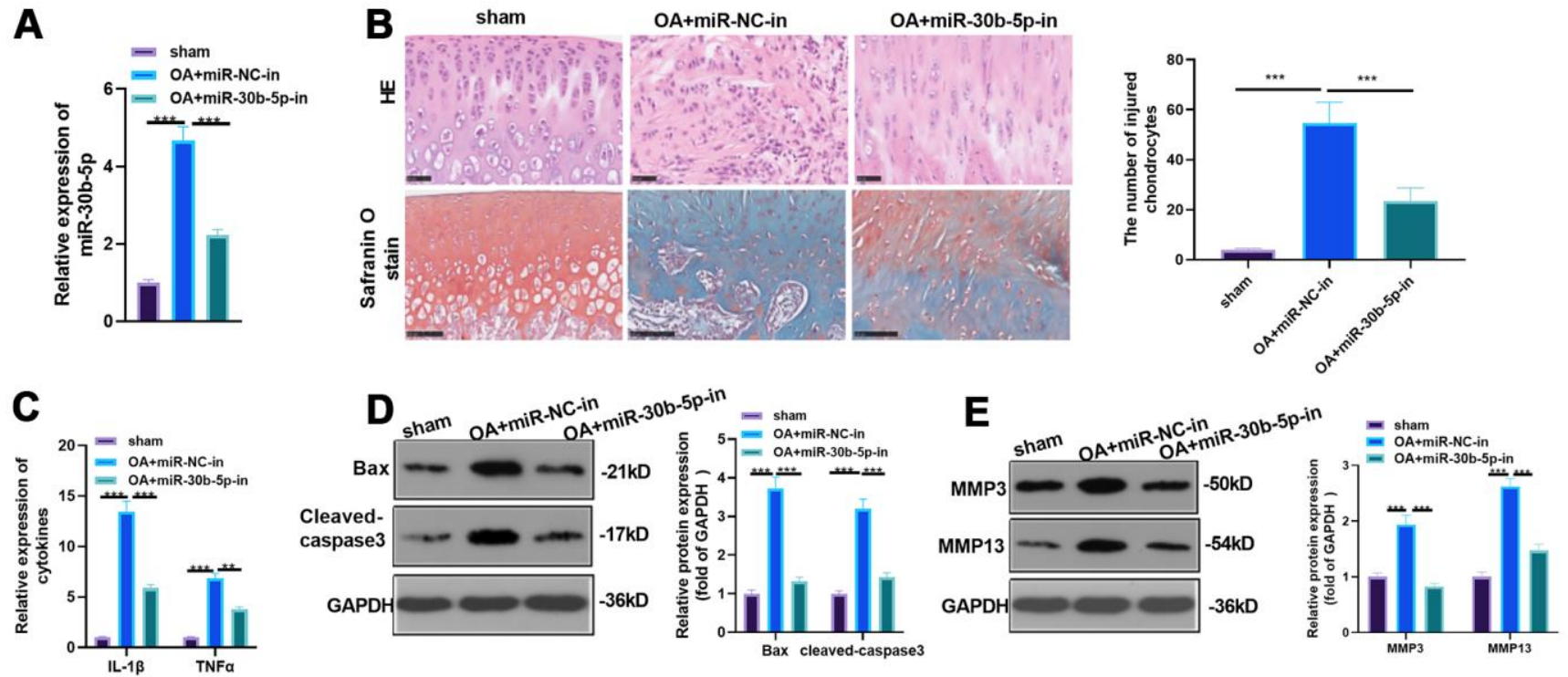

$\mathbf{F}$

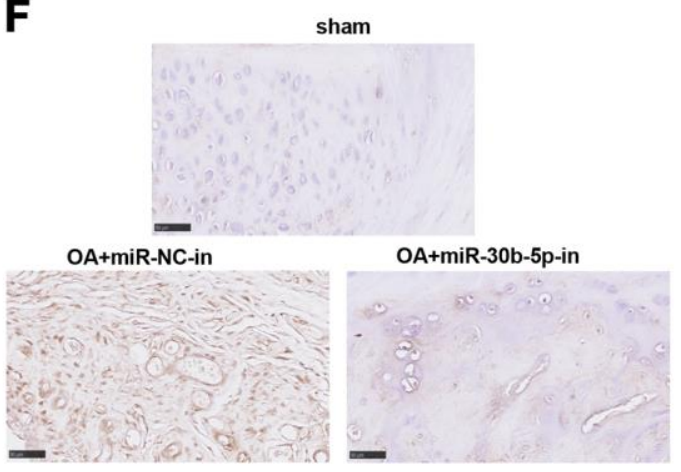

G

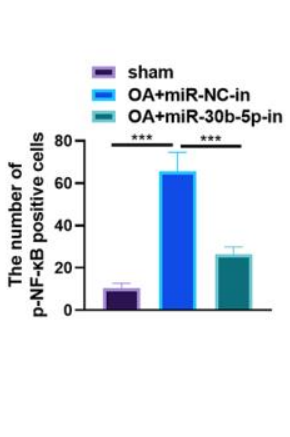

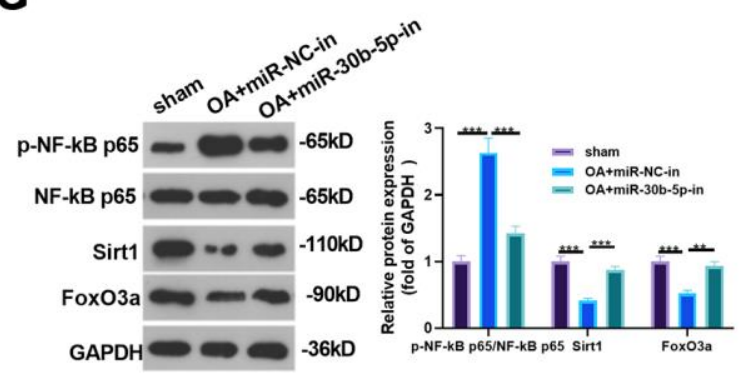

Figure 4. The influence of inhibiting miR-30b-5p on OA rats. miR-30b-5p inhibitors were added to the knee joint cavity of OA rats. (A) The miR-30b-5p profile was measured by RT-qPCR. (B) HE and Safranin O staining were used to observe the morphological differences in cartilage tissue, and the number of injured chondrocytes was counted. (C) The levels of IL-1 $\beta$ and TNF- $\alpha$ were compared by RT-qPCR. (D, E) WB was implemented to test the profiles of Bax, Cleaved-Caspase3, MMP3 and MMP13. (F) The level of NF-kB in the cartilage tissue was gauged by IHC. (G) Expression of NF-KB, SIRT1/FoxO3a in the cartilage tissue was compared by WB. $* * P<0.01$, $* * * P<0.001 . \mathrm{N}=5$. 
transfection led to a higher amount of SIRT1 deposited in the Ago2 antibody group than that in the IgG group, confirming that SIRT1 bound to Ago2 via miR-30b-5p $(P<0.05$, Figure 6D). The expression of SIRT1 and FoxO3a was further checked by RT-qPCR and WB, and it was found that SIRT1 and FoxO3a were evidently down-regulated after the miR-30b-5p mimic transfection $(P<0.05$, Figure $6 \mathrm{E}, 6 \mathrm{~F})$. These results testified that miR-30b-5p bound to SIRT1 and negatively regulated FoxO3a expression.

Overexpressing SIRT1 weakened the miR-30b-5pmediated effect

First, we constructed a SIRT1 overexpression model in HC-A cells and verified the transfection validity
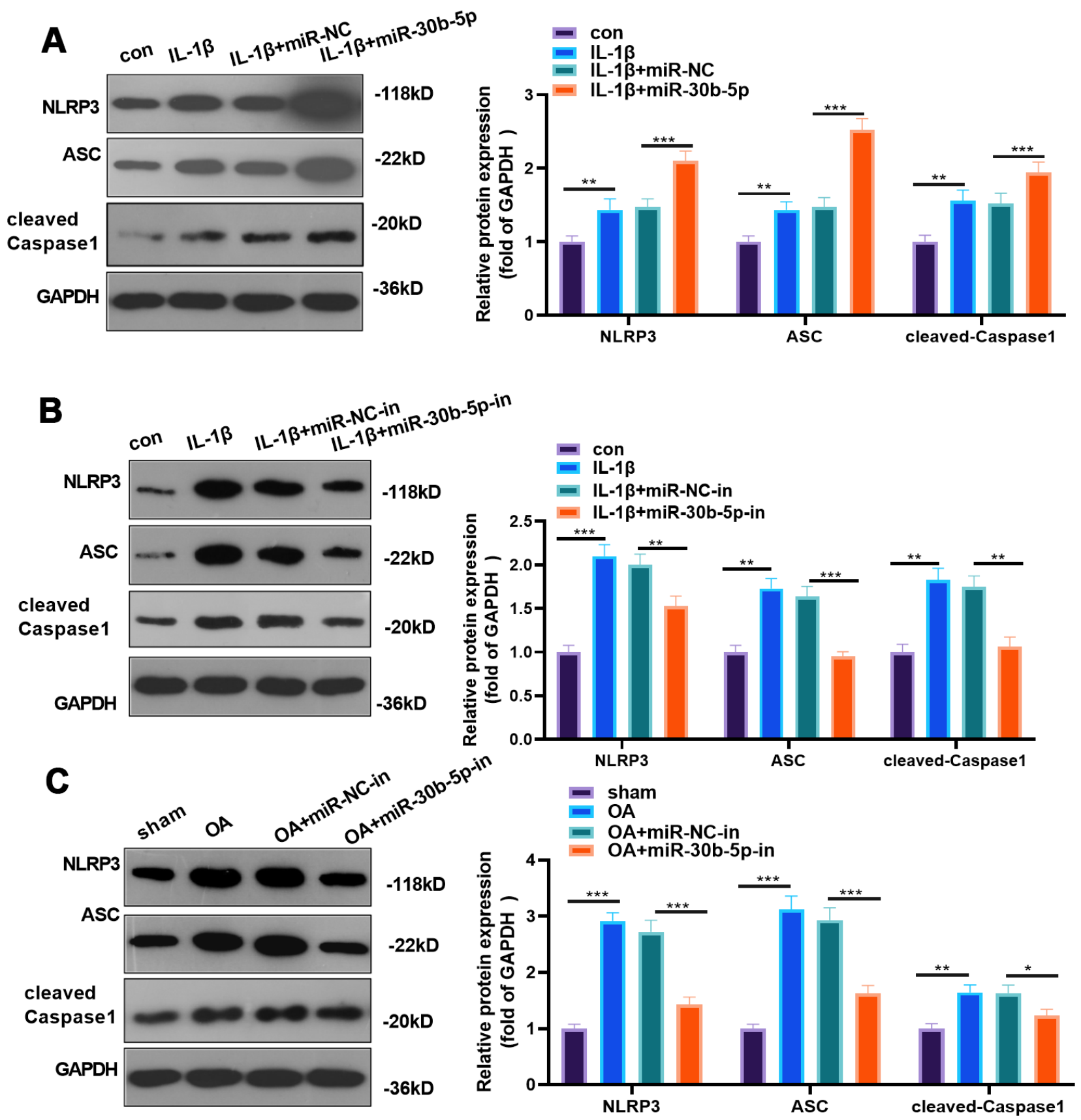

Figure 5. miR-30b-5p facilitated NLRP3 expression in chondrocytes and joint tissues. miR-30b-5p mimics or inhibitors were added to HC-A chondrocytes. (A, B) Protein expression of NLRP3-ASC-cleaved Caspase1 was verified by WB after transfecting miR-30b-5p mimics or inhibitors to IL-1 1 -treated HC-A cells. miR-30b-5p inhibitors were added to the knee joint cavity of OA rats. (C) WB examined the expression of NLRP3-ASC- cleaved Caspase1 in joint tissues after transfecting miR-30b-5p inhibitors. ${ }^{* *} P<0.01, * * * P<0.001$. $\mathrm{N}=5$. 
through RT-qPCR and WB to examine the influence of overexpressing SIRT1 on miR-30b-5p-mediated damaging effects $(P<0.05$, Figure 7A, 7B $)$. Then, IL-1 $\beta$ was given to treat $\mathrm{HC}-\mathrm{A}$ cells, and miR-30b-5p mimics were added to HC-A cells transfected with SIRT1 plasmids. The SIRT1-FoxO3a expression in HC-A cells was testified by RT-qPCR and WB. As a result, SIRT1 and FoxO3a expression was curbed in the IL- $1 \beta+$ miR-30b$5 p+v e c t o r$ group (vs. the IL-1 $\beta$ group). Nevertheless, they were up-regulated after SIRT1 overexpression on the basis of IL-1 $\beta+$ miR-30b-5p+vector treatment $(P<0.05$, Figure $7 \mathrm{C}, 7 \mathrm{D})$. The CCK8 assay results manifested that compared with the IL- $1 \beta$ group, cell viability was weakened after miR-30b-5p overexpression, while overexpressing SIRT1 on this basis promoted chondrocyte viability $(P<0.05$, Figure $7 \mathrm{E})$. Besides, Flow cytometry testified that compared with the IL-1 $\beta$ group, overexpressing miR-30b-5p facilitated cell apoptosis, while overexpressing SIRT1 on this basis abated chondrocyte apoptosis $(P<0.05$, Figure 7F). Moreover, WB results manifested that Bax and Cleaved-Caspase3 were up-regulated after overexpressing miR-30b-5p (vs. the IL- $1 \beta$ group). However, compared with the IL- $1 \beta+$ miR-30b-5p +vector group, Bax and Cleaved-Caspase 3 were significantly downregulated in the IL-1 $\beta+$ miR-30b-5p+SIRT1 group $(P<0.05$, Figure $7 \mathrm{G})$. Furthermore, RT-qPCR results confirmed that the contents of IL- $1 \beta$ and TNF- $\alpha$ were elevated after overexpressing miR-30b-5p (vs. the IL-1 $\beta$ group), while their expression was attenuated by

\section{A}

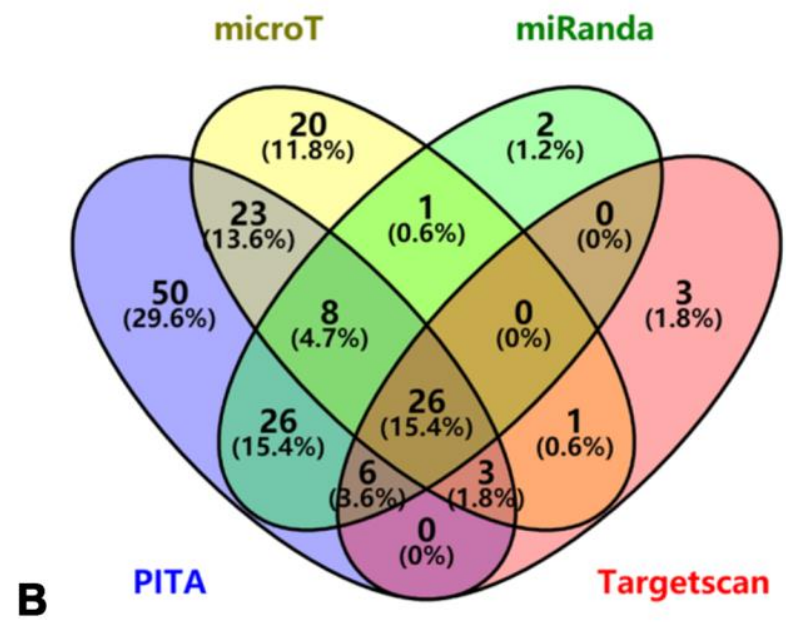

hsa-m $\mathbb{R}-22-3 p \quad$ hsa-m $\mathbb{R}-29 a-3 p$

hsa-m $\mathbb{R}-30 a-5 p \quad$ hsa-m $\mathbb{R}-29 b-3 p$

hsa-m $\mathbb{R}-199 a-5$ p hsa-m $\mathbb{R}-30 c-5 p$

hsa-m $\mathbb{R}-30 d-5$ p hsa-m $\mathbb{R}-199 b-5 p$

hsa-m $\mathbb{R}-212-3$ p hsa-m $\mathbb{R}-30 b-5$ p

hsa-m $\mathbb{R}-128-3$ p hsa-m $\mathbb{R}^{-132-3 p}$

hsa $-m \mathbb{R}-133 a-3$ p hsa-m $\mathbb{R}-135 a-5 p$

hsa-m $\mathbb{R}-138-5$ p hsa-m $\mathbb{R}^{-141-3 p}$

hsa-m $\mathbb{R}-9-5 p \quad$ hsa-m $\mathbb{R}-155-5 p$

hsa-m $\mathbb{R}-29 c-3 p \quad$ hsa-m $\mathbb{R}-200 a-3 p$

hsa-m $\mathbb{R}-30 e^{-}-5$ p hsa-m $\mathbb{R}-135 b-5 p$

hsa-m $\mathbb{R}-133 b$ hsa-m $\mathbb{R}-448$

hsa-m $\mathbb{R}-494-3$ p hsa-m $\mathbb{R}-543$

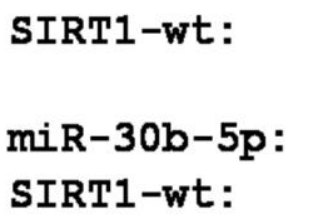

D

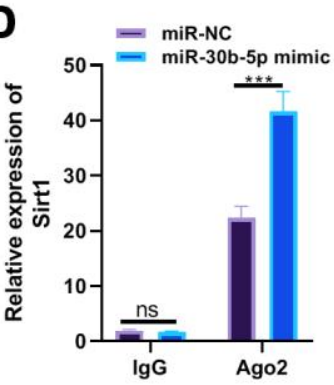

5' caugucazazugAaUgUUUACu $3^{\prime}$ | |||||||| 3' ucgacucacauCCUACAAAUGu 5' $5^{\prime}$ caugucaaaauCAUACAAAUGu $3^{\prime}$

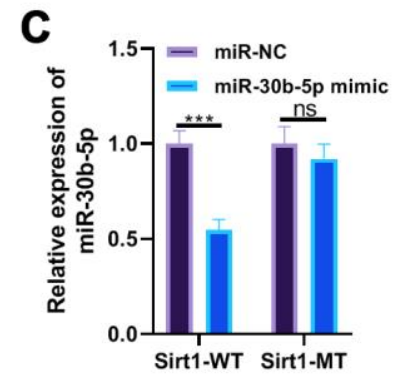

E

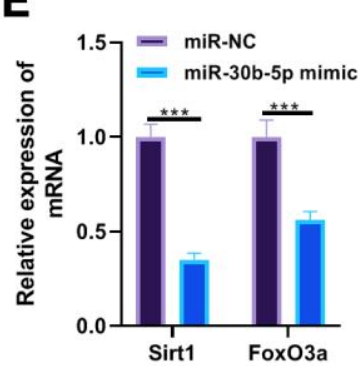

$\mathbf{F}$

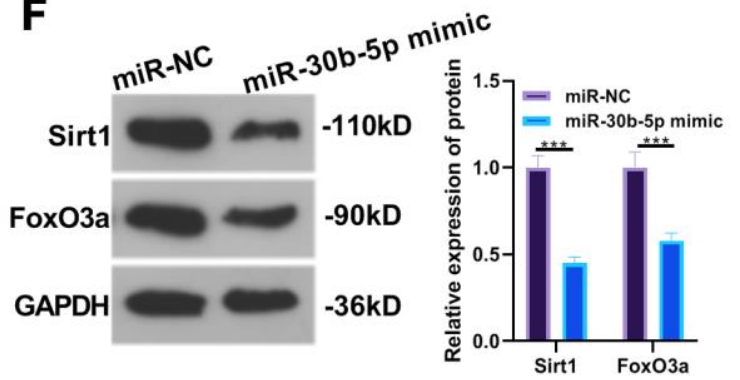

Figure 6. SIRT1 targeted miR-30b-5p. (A, B) The target association between miR-30b-5p and SIRT1 was predicted in the ENCORI database. (C, D) The targeted affinity between miR-30b-5p and SIRT1 was validated by the dual-luciferase reporter assay and RIP experiment, respectively. (E, F) The profiles of SIRT1 and FoxO3a were further evaluated by RT-qPCR and WB. nsP>0.05, $* * * P<0.001$. N=3. 
A

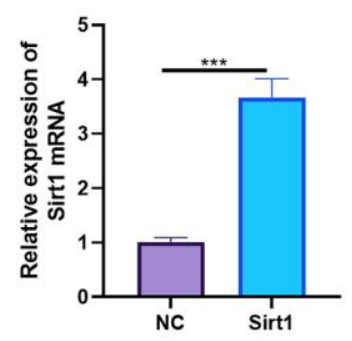

B

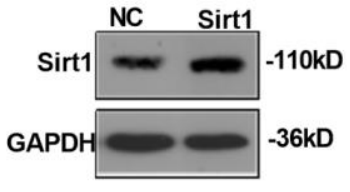

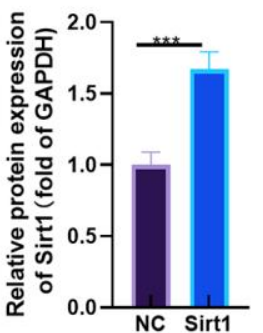

C

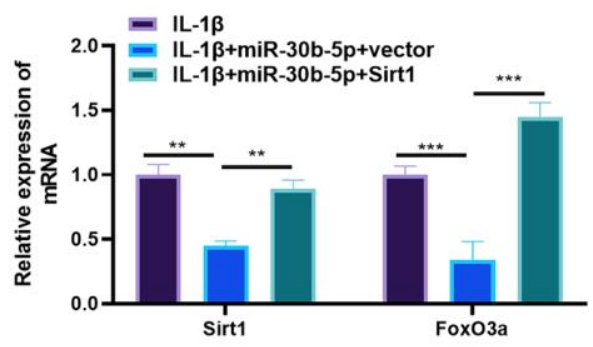

E
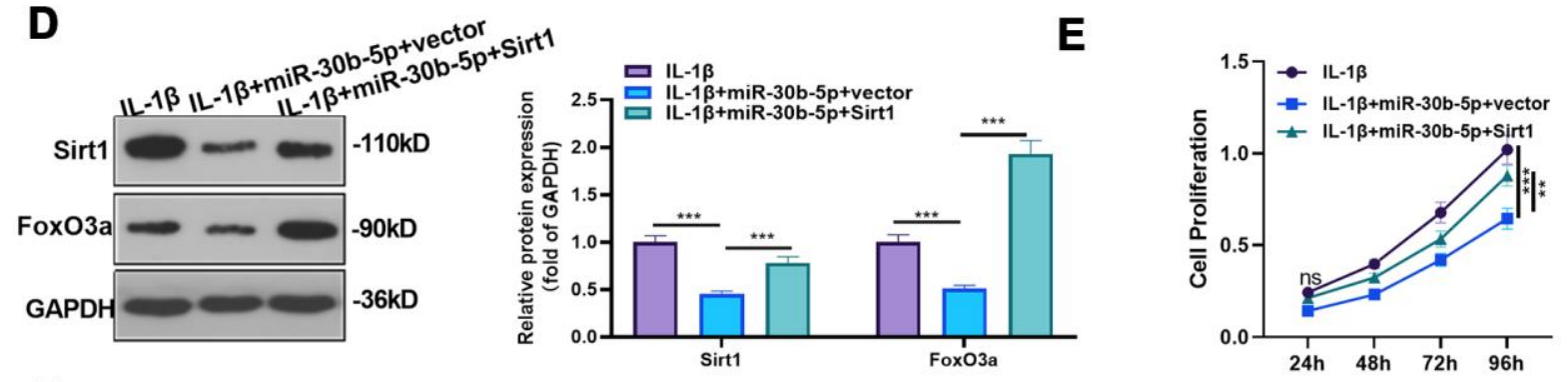

F

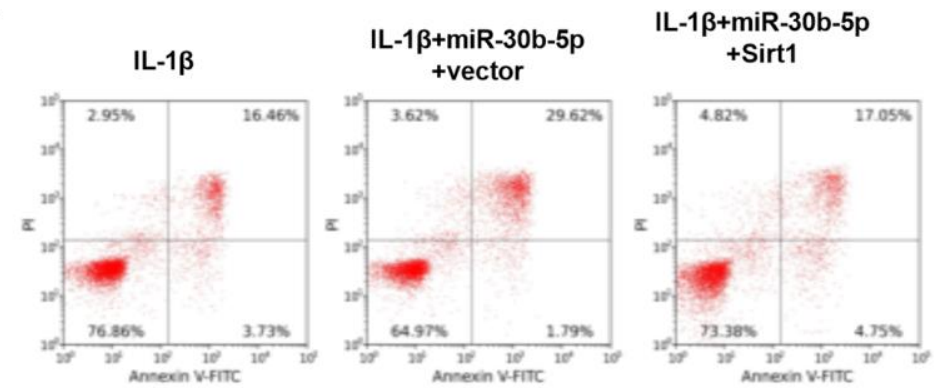

G
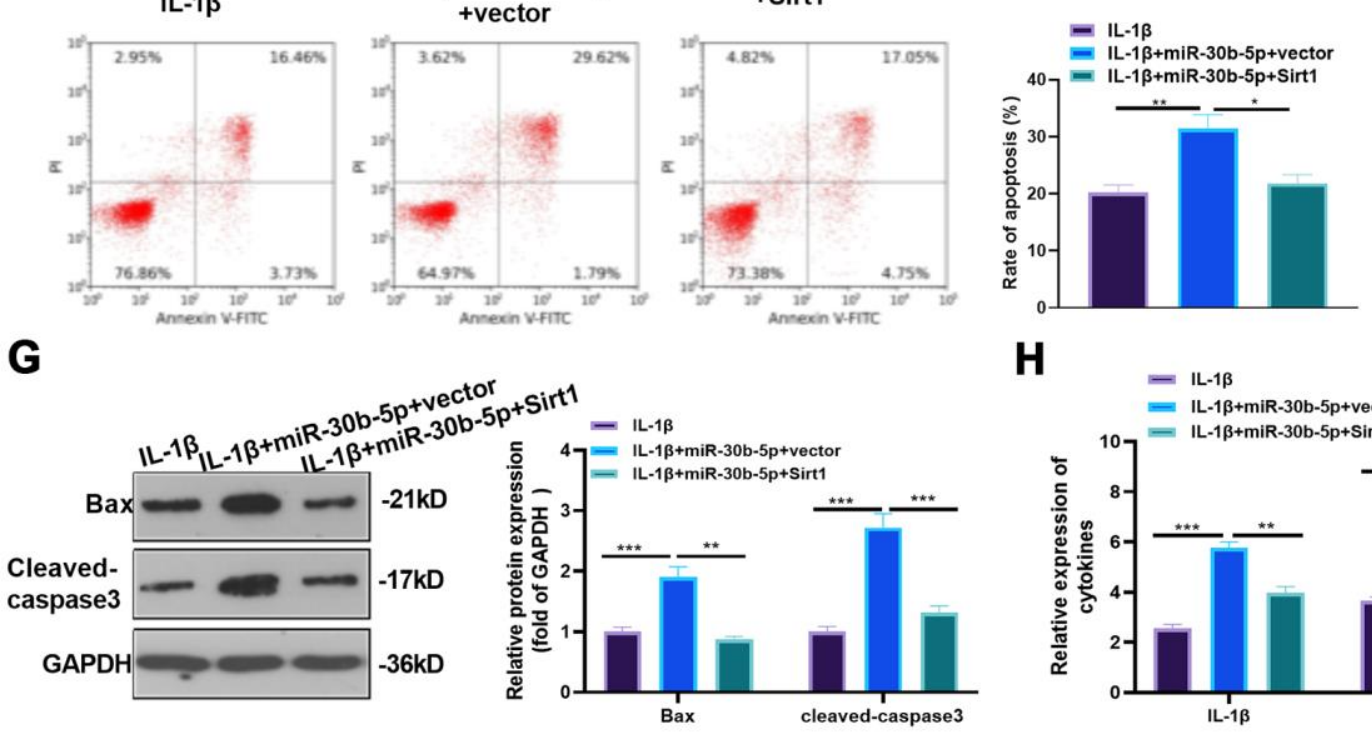

H
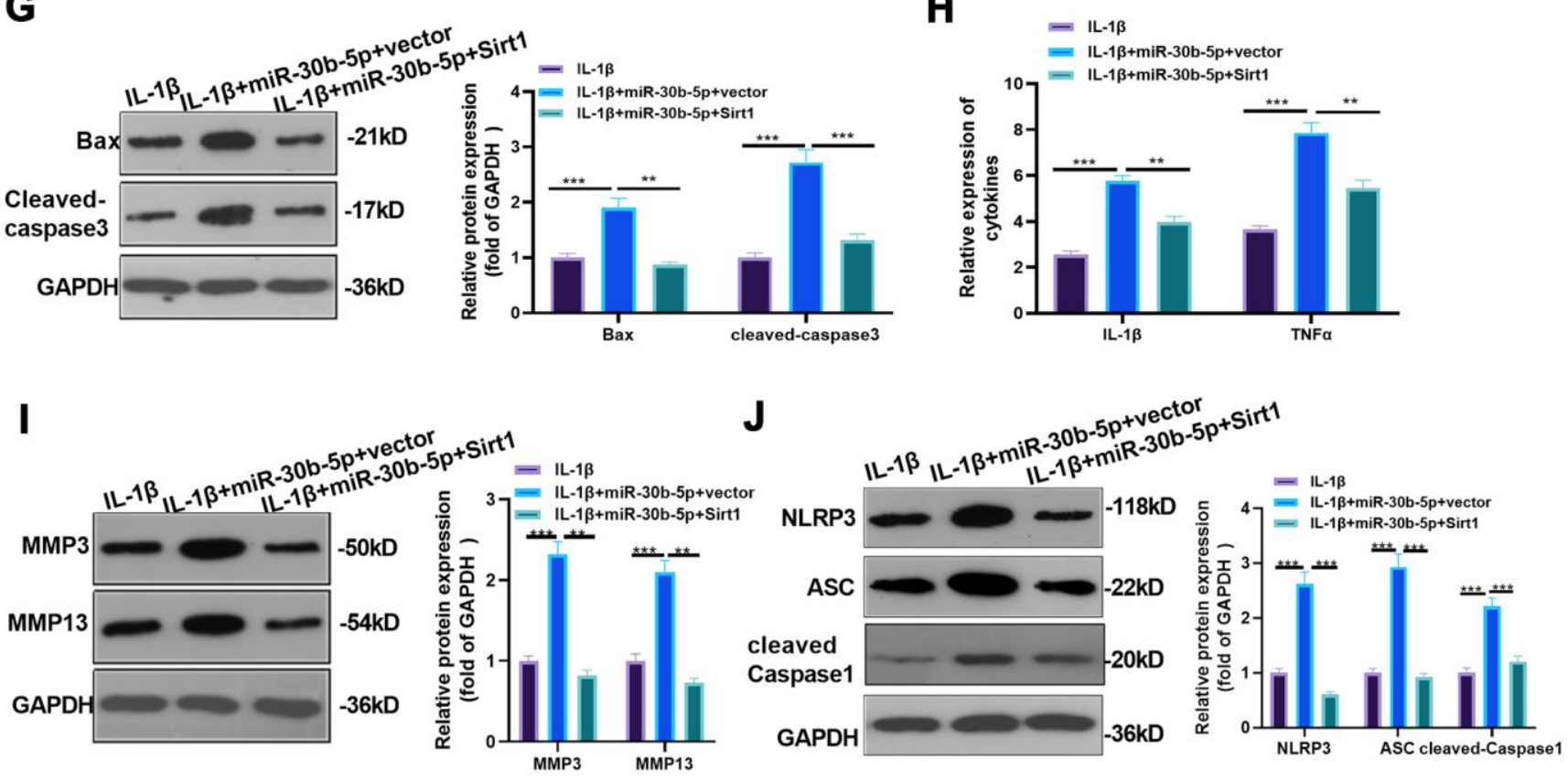

Figure 7. Overexpressing SIRT1 weakened the miR-30b-5p-mediated effect. (A, B) SIRT1 mimics were transfected into IL-1ß-treated HC-A cells, and the transfection validity was verified by RT-qPCR and WB. (C, D) The expression of SIRT1 /FoxO3a in IL-1 $\beta$-treated HC-A cells was assessed by RT-qPCR and WB. (E, F) CCK8 and flow cytometry monitored cell viability and apoptosis, respectively. (G) Profiles of Bax and Cleaved-Caspase3 were measured by WB. (H) RT-qPCR monitored the expression of IL-1 $\beta$ and TNF- $\alpha$. (I, J) The profiles of MMP3, MMP13 and NLRP3-ASC- cleaved Caspase1 were verified by WB. NSP>0.05, $* P<0.05, * * P<0.01, * * * P<0.001 . \mathrm{N}=3$. 
SIRT1 overexpression on this basis $(P<0.05$, Figure $7 \mathrm{H})$. Finally, WB results confirmed that MMP3, MMP13 and NLRP3-ASC-Caspase1 levels were upregulated after overexpressing miR-30b-5p (vs, the IL$1 \beta$ group). In contrast, compared with the IL- $1 \beta+$ miR$30 b-5 p+$ Vector group, these proteins in the IL- $1 \beta+$ miR$30 \mathrm{~b}-5 \mathrm{p}+\mathrm{SIRT} 1$ group were significantly down-regulated $(P<0.05$, Figure 7I, 7J). These experimental results signified that overexpressing SIRT1 in HC-A cells weakened the damage of miR-30b-5p on OA.

\section{Down-regulation of SIRT1 or FoxO3a attenuated the

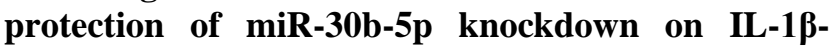 mediated HC-A cells}

To detect the influence of inhibiting SIRT1/FoxO3a on miR-30b-5p knockdown-mediated protective effect, we constructed the low expression model of SIRT1 and FoxO3a in HC-A cells and verified the transfection effect by RT-qPCR and WB $(P<0.05$, Figure $8 \mathrm{~A}-8 \mathrm{D})$. Subsequently, si-SIRT1 or si-FoxO3a was respectively transfected into IL-1 $\beta$-mediated HC-A cells with miR30b-5p knockdown. As the data exhibited, SIRT1 and FoxO3a were up-regulated compared with the IL$1 \beta+$ miR-30b-5p-in group. However, they were downregulated after SIRT1 or FoxO3a knockdown $(P<0.05$, Figure 8E, 8F). CCK8 assay and flow cytometry results showed that knockdown of SIRT1 or FoxO3a impeded the activity of chondrocytes and intensified apoptosis $(P<0.05$, Figure $8 \mathrm{G}, 8 \mathrm{H})$. The expression of IL-1 $\beta$ and $\mathrm{TNF} \alpha$ was significantly decreased after miR-30b-5p inhibition (vs. the IL-1 $\beta$ group). On this basis, the knockdown of SIRT1 or FoxO3a strengthened the profiles of the above-mentioned inflammatory cytokines $(P<0.05$, Figure 8I). WB results testified that the expression of Bax, Cleaved Caspase3, MMP3/MMP13, and NLPRP3-ASC-Caspase1 was curbed in the IL$1 \beta+$ miR-30b-5p-in group (vs. the IL- $1 \beta$ group). Compared with the IL- $1 \beta+$ miR-30b-5p-in group, the expression of the above proteins was boosted after siSIRT1 or si-FoxO3a transfection $\left(P<0.05\right.$, Figure $8 \mathrm{~J}_{-}$ $8 \mathrm{~L})$. These experimental findings indicated that inhibiting SIRT1/FoxO3a in HC-A cells weakened the protective action of miR-30b-5p-in on OA.

\section{Inhibiting NF- $\kappa B$ reduced miR-30b-5p levels and IL- 1ß-mediated HC-A cell injury}

We added BAY 11-7082 to IL-1 $\beta$-treated HC-A cells and given miR-30b-5p mimics for intervention to verify the impact of NF- $\kappa \mathrm{B}$ on the miR-30b-5p expression and IL$1 \beta$-mediated HC-A cell damage. WB and RT-qPCR were implemented to verify the profiles of NF- $\kappa \mathrm{B}$ and miR$30 \mathrm{~b}-5 \mathrm{p}$. Interestingly, compared with the IL- $1 \beta$ group, $\mathrm{NF}-\kappa \mathrm{B}$ and miR-30b-5p were down-regulated after adding BAY 11-7082. However, no significant difference was observed in NF- $\mathrm{BB}$ expression, while miR-30b-5p was up-regulated after the miR-30b-5p mimic treatment (vs. the IL-1 $\beta+$ BAY 11-7082 group) $(P<0.05$, Figure 9A, 9B). Additionally, CCK8 and flow cytometry results exhibited that inhibiting NF- $\mathrm{BB}$ elevated HC-A cell viability and inhibited apoptosis (vs. the IL-1 $\beta$ group). Nevertheless, IL-1 $\beta+$ BAY 11-7082+miR-30b-5p weakened HC-A cell viability and strengthened cell apoptosis (vs. the IL-1 $\beta+\mathrm{BAY} 11-7082$ group) $(P<0.05$, Figure 9C, 9D). Also, WB confirmed that Bax and Cleaved-Caspase 3 were down-regulated after NF- $\kappa \mathrm{B}$ inhibition (vs. the IL$1 \beta$ group), whereas they were significantly up-regulated in the IL-1 $\beta+$ BAY $11-7082+$ miR-30b-5p group (vs. the IL$1 \beta+$ BAY $11-7082$ group) $(P<0.05$, Figure $9 \mathrm{E})$. The expression of IL- $1 \beta$, TNF- $\alpha$, MMP3 and MMP13 was compared by RT-qPCR and WB, respectively. Interestingly, their mRNA profiles were restrained in the $\mathrm{IL}-1 \beta+\mathrm{BAY}$ 11-7082 group (vs. the IL-1 $\beta$ group) and were up-regulated after miR-30b-5p intervention (vs. the IL-1 $\beta$ +BAY 11-7082 group) $(P<0.05$, Figure 9F, 9G). Furthermore, WB and RT-qPCR results uncovered that the profiles of SIRT1 and FoxO3a were up-regulated after $N F-\kappa B$ inhibition compared with the IL- $1 \beta$ group, while they were down-regulated after the miR-30b-5p mimic transfection (vs. the IL-1 $\beta+$ BAY 11-7082 group) $(P<0.05$, Figure 9H, 9I). WB results also manifested that the NLRP3-ASC-Caspase1 inflammasome was downregulated after inhibiting NF- $\mathrm{KB}$ (vs. the IL- $1 \beta$ group), while it was up-regulated after adding miR-30b-5p mimics (vs. the IL-1 $\beta+\mathrm{BAY} 11-7082$ group) $(P<0.05$, Figure 9J). These findings demonstrated that inhibiting $N F-\kappa B$ reduced the miR-30b-5p level, and increasing miR-30b-5p significantly enhanced the injury and inflammation of HC-A cells.

\section{DISCUSSION}

$\mathrm{OA}$ is a frequent chronic orthopedic degenerative disease [22]. Due to the limited understanding of the molecular mechanism of OA, available OA treatments are limited to pain relief or joint replacement [23, 24]. A large number of studies have proved that cytokines and growth factors produced by articular cartilage under the action of mechanical and physical and chemical factors such as trauma, inflammation and infection contribute to OA pathogenesis. They are closely related to the functional changes of the synovial membrane, cartilage, and so on $[25,26]$. In this study, we used surgical DMM and IL- $1 \beta$ to induce in-vivo and in-vitro OA models and found that overexpressing miR-30b-5p accelerated the OA articular cartilage damage by boosting the contents of IL-1 $\beta$, TNF- $\alpha$, MMP3, MMP13 and NLRP3 inflammasomes (Figure 10).

Previous studies have demonstrated that miR-30 contributes to tissue and organ development, tumor 
A

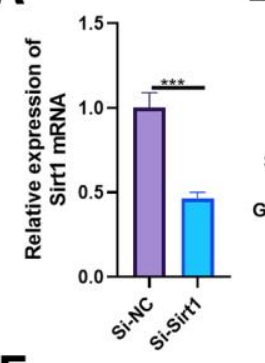

E

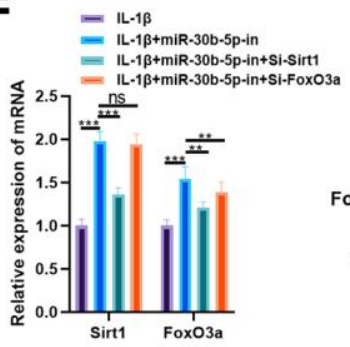

H
B

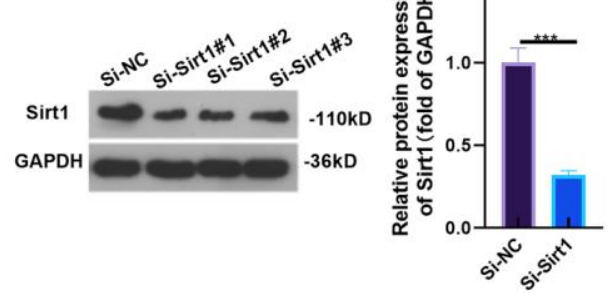

C

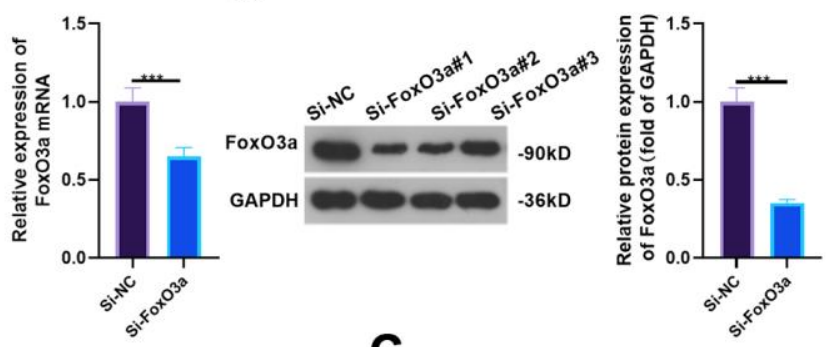

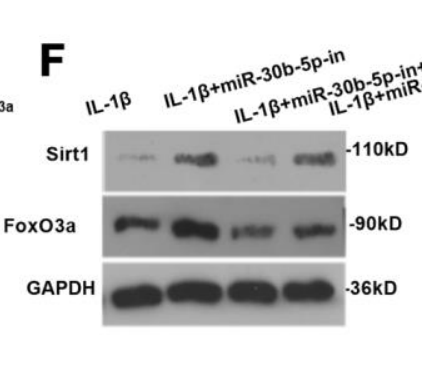

sitt

is For

3a $=$

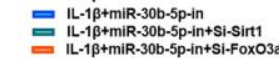

G

$\rightarrow$ แ

- IL-1 $\beta+m i R-30 b-5 p-i n$

\# IL-1 $\beta+$ miR-30b-5p-in+Si-Sirt1
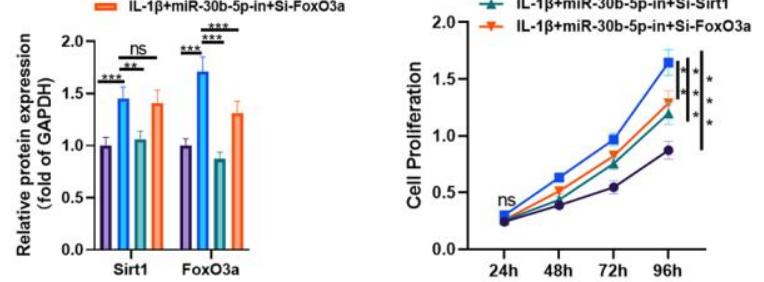

I

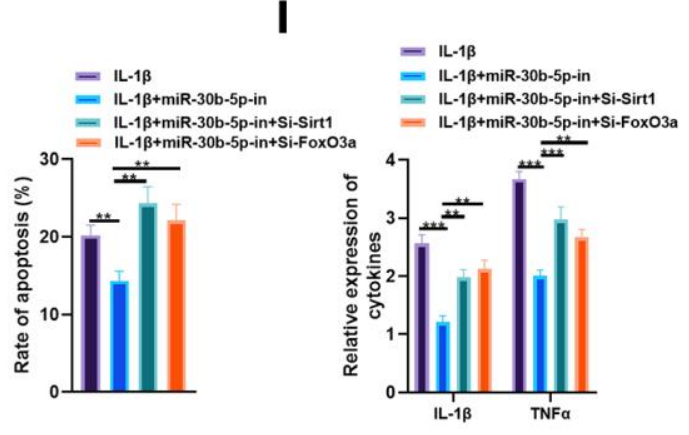

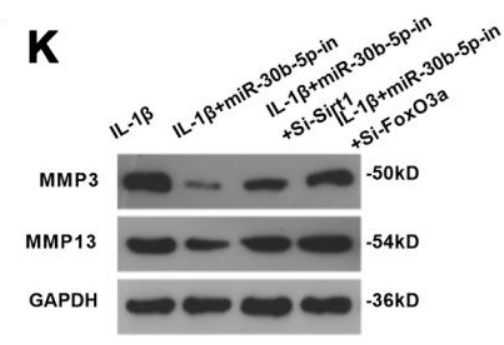

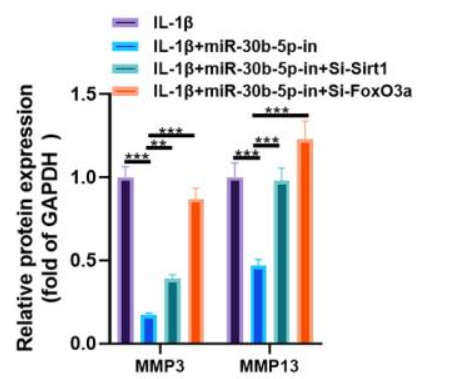

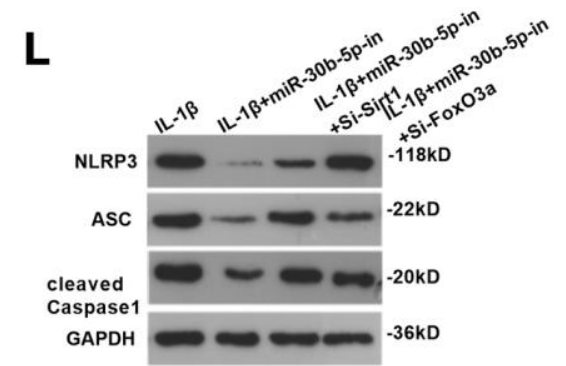

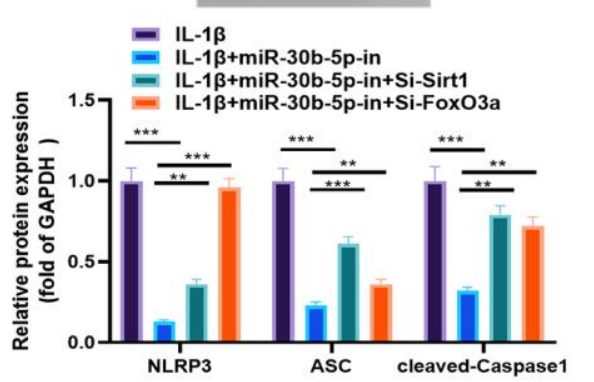

Figure 8. Inhibiting SIRT1/FoxO3a curbed the protection of miR-30b-5p knockdown on IL-1ß-mediated HC-A cells. (A-D) siSIRT1 and si-FoxO3a were transfected into IL-1 $\beta$-treated HC-A cells and their transfection effects were verified by RT-qPCR and WB. (E, F) The SIRT1/FoxO3a expression in IL-1B-treated HC-A cells was checked by RT-qPCR and WB, respectively. (G, H) The CCK8 method and flow cytometry were utilized to gauge cell viability and apoptosis. (I) The levels of IL-1 $\beta$ and TNF $\alpha$ were monitored by RT-qPCR. (J-L) The expression of Bax, cleaved Caspase3, MMP3, MMP13 and NLRP3-ASC-cleaved Caspase1 was examined by WB. nsP $>0.05,{ }^{* * P}<0.01$, $* * * P<0.001 . \mathrm{N}=3$. 
progression, and inflammatory injury [27]. Some studies have shown that miR-30 family members miR30b, miR-30c, miR-30d and miR-30e are reversely related to Runx2 expression under mechanical unloading conditions, which impede osteoblast differentiation by repressing Runx2 in MC3T3-E1 cells [28]. Additionally, IncRNA MALAT1 heightens Runx2 expression and osteoblastic differentiation of fat- derived mesenchymal stem cells by targeting and dampening miR-30 [29]. miR-30b-5p is a member of the miR-30 family, which is located at 8q24.2 and $88 \mathrm{bp}$ long. It is reported that miR-30b-5p exerts crucial function in myocardial hypertrophy [30], ischemic myocardial injury [31], osteosarcoma [32], bone metastasis of prostate cancer [33], and other diseases. Over the years, the function of miR-30b-5p in
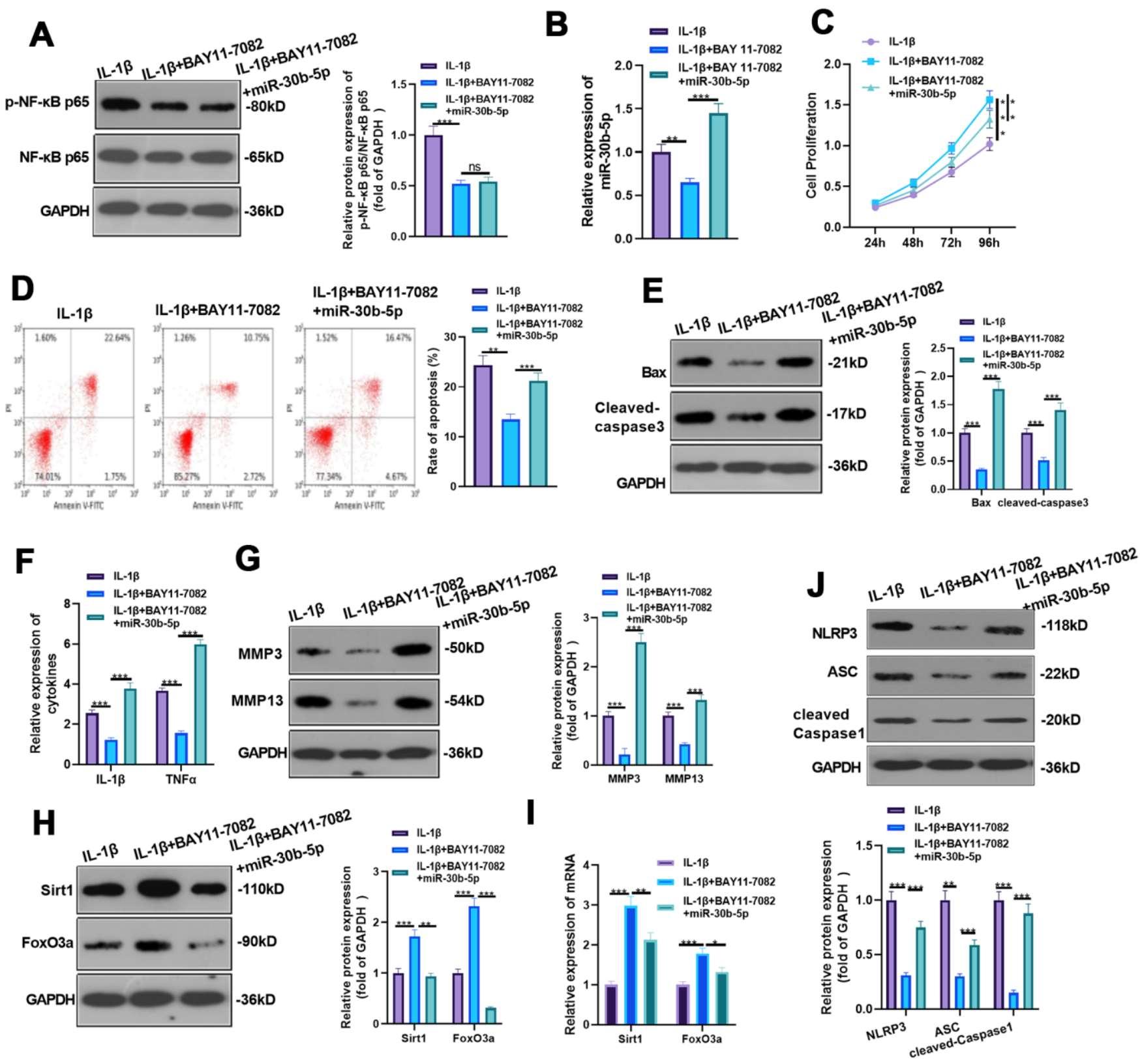

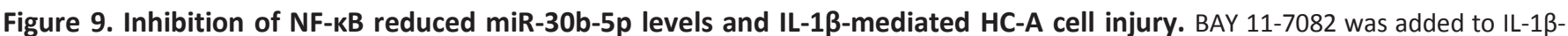
mediated HC-A cells and miR-30b-5p mimics were given. (A, B) NF-KB and miR-30b-5p expression was monitored by WB and RT-qPCR, respectively. (C, D) Cell proliferation and apoptosis were determined by CCK8 and flow cytometry, respectively. (E) Expression of Bax and Cleaved-Caspase3 was determined by WB. (F, G) RT-GPCR and WB were conducted to compare the levels of IL-1 $\beta$, TNF- $\alpha$, MMP3 and MMP13. (H, I) The expression of SIRT1 and FoxO3a was assessed by RT-qPCR. (J) WB detected the expression of NLRP3-ASC-cleaved Caspase1 inflammasomes. ${ }^{* *} P<0.01,{ }^{* * *} P<0.001 . \mathrm{N}=3$. 
inflammatory diseases has also been identified. For example, $\mathrm{Li} \mathrm{Z}$ et al. found that IncRNA DLEU1 upregulates SRPK1 by binding with miR-30b-5p, which has substantial value in regulating the spinal cord inflammation in chronic constriction injury-induced rats and mediating the hypersensitivity of neuropathic pain [34]. More importantly, Wang Q et al. discovered that miR-30b-5p mediates postmenopausal osteoporosis evolvement. On the other hand, lncRNA MEG3 attenuates the osteogenic differentiation of bone marrow mesenchymal stem cells from postmenopausal osteoporosis by targeting miR-30b-5p [35]. Here, we also discovered that the miR-30b-5p profile was heightened and positively linked with pro-inflammatory responses in OA patients' articular fluid, OA rats' articular cartilage tissues, and IL- $1 \beta$-treated chondrocytes. Meanwhile, overexpressing miR-30b-5p significantly enhanced IL- $1 \beta$-mediated chondrocyte apoptosis and inflammation and up-regulated IL-1 $\beta$, TNF- $\alpha$ and NLRP3 inflammasomes. Oppositely, knocking down miR-30b-5p showed the reverse effect, which was testified in vivo. These findings confirmed that miR-30b-5p expedited OA evolvement.
SIRT1 is a highly conserved deacetylase dependent on NAD+ [36]. Foxo is a fork protein family subgroup, and FoxO3a is a vital member of the Foxo family. It has been found that FoxO3a expression and deacetylation are both regulated by SIRT1, and the expression and activation of the SIRT1-FoxO3a signaling pathway are involved in regulating DNA damage and repair, modulating cell differentiation, and reducing inflammation, oxidative damage, vascular diseases, and osteoporosis [37, 38]. For instance, Tseng PC et al. reported that resveratrol up-regulates RUNX2 through the SIRT1/FoxO3a axis to promote human mesenchymal stem cell osteogenesis [39]. Several studies manifested that overexpressing SIRT1 in mesenchymal stem cells facilitates the bone formation of osteoblasts through deacetylation of FoxO3a and inhibition of oxidative stress, thereby alleviating osteoporosis [40].

At the same time, Liang $\mathrm{W}$ et al. claimed that the SIRT1/FoxO3a axis is a potential target for osteoporosis treatment, which suggests that the SIRT1/FoxO3a contributes to orthopedic diseases [41]. Consistent with

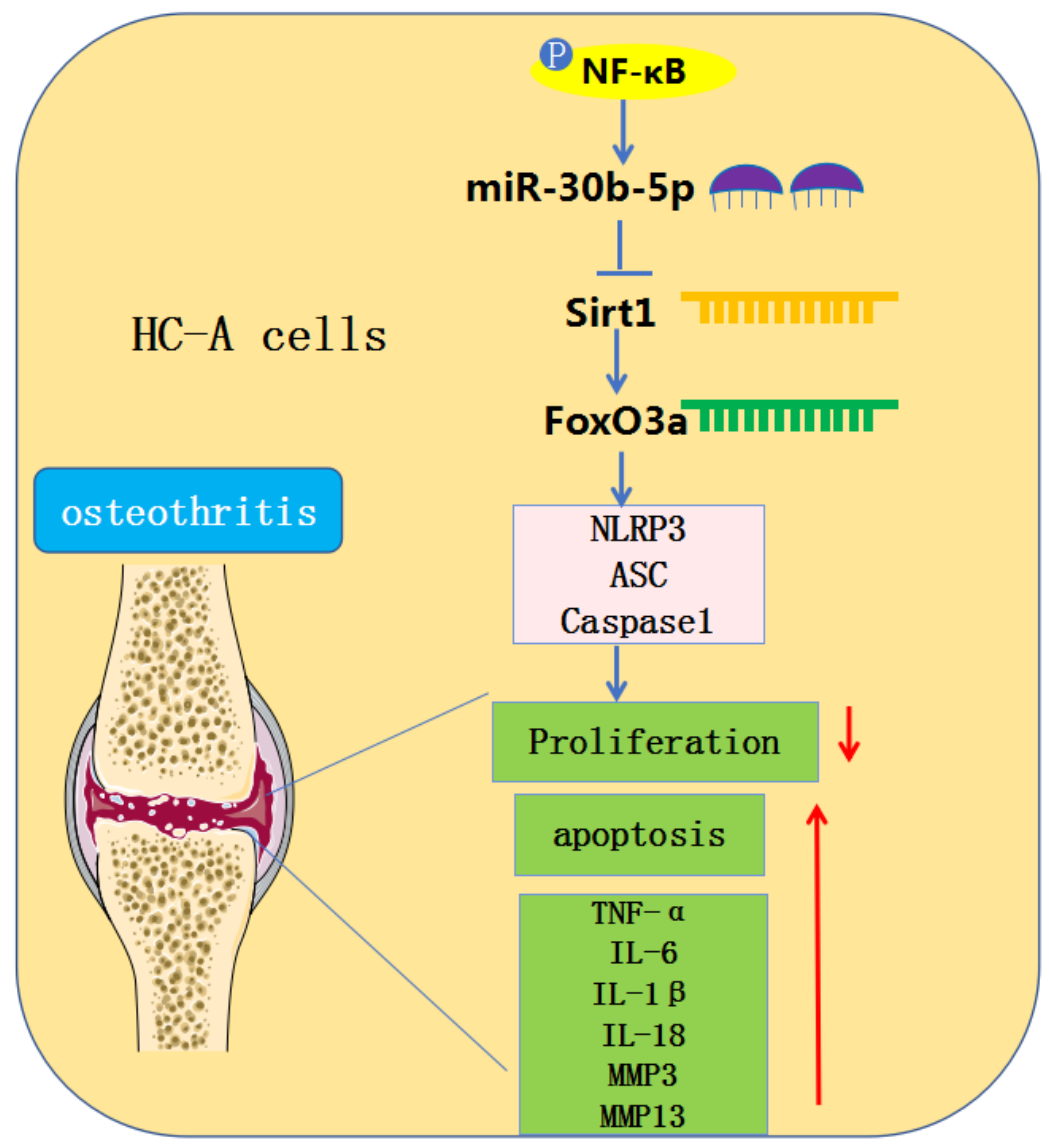

Figure 10. Graphical abstract. In OA chondrocytes, NF-KB-mediated miR-30b-5p activates NLRP3 inflammasomes by targeting and abating the SIRT1/FoxO3a expression, thereby aggravating joint pain and articular cartilage damage in OA patients. 
the above research, this article revealed that miR-30b$5 \mathrm{p}$ bound to SIRT1 and repressed its expression, and overexpressing miR-30b-5p inactivated SIRT1 and FoxO3a. Moreover, the rescue experiment illustrated that in IL-1 $\beta$-treated HC-A chondrocytes, overexpressing SIRT1 significantly reduced the damage of miR-30b-5p to articular cartilage, while knocking down SIRT1 or FoxO3a reversed miR-30b-5p-in-mediated anti-inflammatory and anti-apoptosis effects. These findings confirmed that miR-30b-5p exerts its damaging effect on articular cartilage by inhibiting the SIRT1/FoxO3a expression, while overexpressing SIRT1 prevented against OA.

As one of the classical signaling pathways, TLR4/NF- $\mathrm{BB}$ is extensively studied in inflammation [42]. NF- $\kappa B$ activation has been shown to directly lead to NLRP3 inflammasome activation, and NLRP3 is of important value in the development of inflammatory diseases, especially OA [43, 44]. This article expounded that overexpressing miR-30b-5p facilitated the NLRP3 level in OA rat articular cartilage tissues and IL- $1 \beta$-treated HCA chondrocytes and mediated chondrocyte apoptosis, which was also consistent with the results of Fioravanti et al. [45]. More importantly, NF- $\kappa B$ activation leads to increased miR-30b-5p, while inhibition of NF- $\kappa B$ downregulates miR-30b-5p, boosts HC-A cell viability, and suppresses apoptosis. Besides, miR-30b-5p targets SIRT1-FoxO3a and activates NLRP3-. This study found a new way to activate NLRP3. Namely, inhibiting NF- $\kappa B$ inactivates the NLRP3 pathway and alleviates OA by regulating miR-30b-5p.

Overall, this article confirmed that the miR-30b-5p profile was heightened in the articular cartilage tissues and $\mathrm{HC}-\mathrm{A}$ chondrocytes of $\mathrm{OA}$ patients. Meanwhile, NF- $\kappa B$-mediated miR-30b-5p induces NLPR3 activation by targeting the SIRT1/FoxO3a axis, thus promoting joint pain and articular cartilage damage in OA patients (Figure 10). This research offers a referable molecular mechanism for studying OA pathogenesis, while more clinical samples are needed to figure out the expression characteristics and effects of miR-30b-5p in clinical trials.

\section{AUTHOR CONTRIBUTIONS}

Conceived and designed the experiments: Xiaoyang Li; Chao Zheng Performed the experiments: Jie Zhang; Haiting Xu. Statistical analysis: Xiaoming Shi; Haiting Xu. Wrote the paper: Jie Zhang; Xiaoyang Li. All authors read and approved the final manuscript.

\section{CONFLICTS OF INTEREST}

The authors declare that they have no conflicts of interest.

\section{FUNDING}

This research did not receive any specific grant from funding agencies in the public, commercial, or not- forprofit sectors.

\section{Editorial note}

\& This corresponding author has a verified history of publications using a personal email address for correspondence.

\section{REFERENCES}

1. Geyer M, Schönfeld C. Novel Insights into the Pathogenesis of Osteoarthritis. Curr Rheumatol Rev. 2018; 14:98-107. https://doi.org/10.2174/157339711366617080712231 2 PMID:28782470

2. Lambova SN, Müller-Ladner U. Osteoarthritis - Current Insights in Pathogenesis, Diagnosis and Treatment. Curr Rheumatol Rev. 2018; 14:91-97. https://doi.org/10.2174/157339711402180706144757 PMID: $\underline{30003854}$

3. Flemming DJ, Gustas-French CN. Rapidly Progressive Osteoarthritis: a Review of the Clinical and Radiologic Presentation. Curr Rheumatol Rep. 2017; 19:42. https://doi.org/10.1007/s11926-017-0665-5 PMID:28689367

4. Lieberthal J, Sambamurthy N, Scanzello CR. Inflammation in joint injury and post-traumatic osteoarthritis. Osteoarthritis Cartilage. 2015; 23: 1825-34.

https://doi.org/10.1016/i.joca.2015.08.015 PMID:26521728

5. Luo C, Liang JS, Gong J, Zhang HL, Feng ZJ, Yang HT, Zhang $\mathrm{HB}$, Kong QH. The function of microRNA-34a in osteoarthritis. Bratisl Lek Listy. 2019; 120:386-91. https://doi.org/10.4149/BLL 2019063 PMID:31113203

6. Li F, Yao J, Hao Q, Duan Z. miRNA-103 promotes chondrocyte apoptosis by down-regulation of Sphingosine kinase-1 and ameliorates PI3K/AKT pathway in osteoarthritis. Biosci Rep. 2019; 39:BSR20191255.

https://doi.org/10.1042/BSR20191255

PMID:31652455

7. Wei C, Li L, Gupta S. NF-KB-mediated miR-30b regulation in cardiomyocytes cell death by targeting Bcl-2. Mol Cell Biochem. 2014; 387:135-41. https://doi.org/10.1007/s11010-013-1878-1 PMID:24178239 
8. Yang L, Tian L, Zhang Z, Zhou X, Ji X, Liu F, Dong C, Hou L, Zhao X, Chang N, Yang L, Li L. Cannabinoid Receptor 1/miR-30b-5p Axis Governs Macrophage NLRP3 Expression and Inflammasome Activation in Liver Inflammatory Disease. Mol Ther Nucleic Acids. 2020; 20:725-38.

https://doi.org/10.1016/i.omtn.2020.04.010

PMID:32408051

9. Wang $Y Q$, Cao $Q$, Wang F, Huang LY, Sang $T$, Liu F, Chen SY. SIRT1 Protects Against Oxidative StressInduced Endothelial Progenitor Cells Apoptosis by Inhibiting FOXO3a via FOXO3a Ubiquitination and Degradation. J Cell Physiol. 2015; 230:2098-107. https://doi.org/10.1002/jcp.24938 PMID:25640014

10. Olmos Y, Sánchez-Gómez FJ, Wild B, García-Quintans N, Cabezudo S, Lamas S, Monsalve M. SirT1 regulation of antioxidant genes is dependent on the formation of a FoxO3a/PGC-1 $\alpha$ complex. Antioxid Redox Signal. 2013; 19:1507-21.

https://doi.org/10.1089/ars.2012.4713

PMID:23461683

11. Yan S, Wang $M$, Zhao J, Zhang $H$, Zhou C, Jin L, Zhang $Y$, Qiu X, Ma B, Fan Q. MicroRNA-34a affects chondrocyte apoptosis and proliferation by targeting the SIRT1/p53 signaling pathway during the pathogenesis of osteoarthritis. Int J Mol Med. 2016; 38:201-09.

https://doi.org/10.3892/ijmm.2016.2618 PMID:27247228

12. Chen H, Qi J, Bi Q, Zhang S. Suppression of miR-301a alleviates LPS-induced inflammatory injury in ATDC5 chondrogenic cells by targeting Sirt1. Int J Clin Exp Pathol. 2017; 10:8991-9000.

PMID:31966769

13. Bai Y, Chen K, Zhan J, Wu M. miR-122/SIRT1 axis regulates chondrocyte extracellular matrix degradation in osteoarthritis. Biosci Rep. 2020; 40:BSR20191908.

https://doi.org/10.1042/BSR20191908

PMID: $\underline{32395770}$

14. Song L, Pei L, Yao S, Wu Y, Shang Y. NLRP3 Inflammasome in Neurological Diseases, from Functions to Therapies. Front Cell Neurosci. 2017; 11:63.

https://doi.org/10.3389/fncel.2017.00063 PMID:28337127

15. McAllister MJ, Chemaly M, Eakin AJ, Gibson DS, McGilligan VE. NLRP3 as a potentially novel biomarker for the management of osteoarthritis. Osteoarthritis Cartilage. 2018; 26:612-19.

https://doi.org/10.1016/j.joca.2018.02.901

PMID:29499288

16. Dong HC, Li PN, Chen CJ, Xu X, Zhang H, Liu G, Zheng L, Li P. Sinomenine Attenuates Cartilage Degeneration by Regulating miR-223-3p/NLRP3 Inflammasome
Signaling. Inflammation. 2019; 42:1265-75. https://doi.org/10.1007/s10753-019-00986-3 PMID:30847744

17. Qian K, Zhang L, Shi K. Triptolide prevents osteoarthritis via inhibiting hsa-miR-20b. Inflammopharmacology. 2019; 27:109-19. https://doi.org/10.1007/s10787-018-0509-6 PMID:29974310

18. Glasson SS, Blanchet TJ, Morris EA. The surgical destabilization of the medial meniscus (DMM) model of osteoarthritis in the 129/SvEv mouse. Osteoarthritis Cartilage. 2007; 15:1061-69.

https://doi.org/10.1016/i.joca.2007.03.006 PMID: 17470400

19. Rogart JN, Barrach HJ, Chichester CO. Articular collagen degradation in the Hulth-Telhag model of osteoarthritis. Osteoarthritis Cartilage. 1999; 7:539-47. https://doi.org/10.1053/joca.1999.0258 PMID:10558852

20. Li YF, Li SH, Liu Y, Luo YT. Long Noncoding RNA CIR Promotes Chondrocyte Extracellular Matrix Degradation in Osteoarthritis by Acting as a Sponge For Mir-27b. Cell Physiol Biochem. 2017; 43:602-10. https://doi.org/10.1159/000480532 PMID:28934732

21. Huang Z, Yang R, Zhang L, Zhu M, Zhang C, Wen J, Li H. BRD4 inhibition alleviates mechanical stress-induced TMJ OA-like pathological changes and attenuates TREM1-mediated inflammatory response. Clin Epigenetics. 2021; 13:10.

https://doi.org/10.1186/s13148-021-01008-6

PMID:33446277

22. Glyn-Jones S, Palmer AJ, Agricola R, Price AJ, Vincent TL, Weinans H, Carr AJ. Osteoarthritis. Lancet. 2015; 386:376-87.

https://doi.org/10.1016/S0140-6736(14)60802-3 PMID:25748615

23. Rychel JK. Diagnosis and treatment of osteoarthritis. Top Companion Anim Med. 2010; 25:20-25. https://doi.org/10.1053/i.tcam.2009.10.005 PMID:20188335

24. Saccomano SJ. Osteoarthritis treatment: Decreasing pain, improving mobility. Nurse Pract. 2018; 43:49-55. https://doi.org/10.1097/01.NPR.0000544281.05010.86 PMID:30134438

25. O'Neill TW, Felson DT. Mechanisms of Osteoarthritis (OA) Pain. Curr Osteoporos Rep. 2018; 16:611-16. https://doi.org/10.1007/s11914-018-0477-1 PMID: $\underline{30155845}$

26. Hwang HS, Kim HA. Chondrocyte Apoptosis in the Pathogenesis of Osteoarthritis. Int J Mol Sci. 2015; 16:26035-54. 
https://doi.org/10.3390/ijms161125943

PMID:26528972

27. Mao L, Liu S, Hu L, Jia L, Wang H, Guo M, Chen C, Liu Y, $\mathrm{Xu}$ L. miR-30 Family: A Promising Regulator in Development and Disease. Biomed Res Int. 2018; 2018:9623412.

https://doi.org/10.1155/2018/9623412

PMID: 30003109

28. Zhang L, Li G, Wang K, Wang Y, Dong J, Wang H, Xu L, Shi F, Cao X, Hu Z, Zhang S. MiR-30 family members inhibit osteoblast differentiation by suppressing Runx2 under unloading conditions in MC3T3-E1 cells. Biochem Biophys Res Commun. 2020; 522:164-70. https://doi.org/10.1016/j.bbrc.2019.11.057 PMID:31757419

29. Yi J, Liu D, Xiao J. LncRNA MALAT1 sponges miR-30 to promote osteoblast differentiation of adipose-derived mesenchymal stem cells by promotion of Runx2 expression. Cell Tissue Res. 2019; 376:113-21.

https://doi.org/10.1007/s00441-018-2963-2 PMID:30511267

30. Zhu YF, Wang R, Chen W, Cao YD, Li LP, Chen X. miR133a-3p attenuates cardiomyocyte hypertrophy through inhibiting pyroptosis activation by targeting IKKE. Acta Histochem. 2021; 123:151653.

https://doi.org/10.1016/i.acthis.2020.151653 PMID:33246224

31. Li M, Ding W, Tariq MA, Chang W, Zhang X, Xu W, Hou $L$, Wang $Y$, Wang J. A circular transcript of $n c x 1$ gene mediates ischemic myocardial injury by targeting miR133a-3p. Theranostics. 2018; 8:5855-69. https://doi.org/10.7150/thno.27285 PMID:30613267

32. Qu Z, Li S. Long noncoding RNA LINC01278 favors the progression of osteosarcoma via modulating miR-133a3p/PTHR1 signaling. J Cell Physiol. 2020. [Epub ahead of print].

https://doi.org/10.1002/jcp.29582

PMID:31994731

33. Zhang $Y$, Dun $Y$, Zhou S, Huang XH. LncRNA HOXD-AS1 promotes epithelial ovarian cancer cells proliferation and invasion by targeting miR-133a-3p and activating $\mathrm{Wnt} / \beta$-catenin signaling pathway. Biomed Pharmacother. 2017; 96:1216-21.

https://doi.org/10.1016/i.biopha.2017.11.096 PMID:29239819

34. Li Z, Li A, Yan L, Yang T, Xu W, Fan P. Downregulation of long noncoding RNA DLEU1 attenuates hypersensitivity in chronic constriction injury-induced neuropathic pain in rats by targeting miR-133a-3p/SRPK1 axis. Mol Med. 2020; 26:104. https://doi.org/10.1186/s10020-020-00235-6 PMID:33167866
35. Wang $Q$, Li $Y$, Zhang $Y$, Ma L, Lin L, Meng J, Jiang L, Wang L, Zhou P, Zhang Y. LncRNA MEG3 inhibited osteogenic differentiation of bone marrow mesenchymal stem cells from postmenopausal osteoporosis by targeting miR-133a-3p. Biomed Pharmacother. 2017; 89:1178-86.

https://doi.org/10.1016/i.biopha.2017.02.090 PMID:28320084

36. Tang BL. Sirt1 and the Mitochondria. Mol Cells. 2016; 39:87-95.

https://doi.org/10.14348/molcells.2016.2318 PMID:26831453

37. Ham HJ, Park JW, Bae YS. Defect of SIRT1-FoxO3a axis is associated with the production of reactive oxygen species during protein kinase CK2 downregulationmediated cellular senescence and nematode aging. BMB Rep. 2019; 52:265-70.

https://doi.org/10.5483/BMBRep.2019.52.4.156 PMID: $\underline{30103847}$

38. Zhao $X$, Liu Y, Zhu G, Liang $Y$, Liu B, Wu Y, Han M, Sun W, Han $Y$, Chen G, Jiang J. SIRT1 downregulation mediated Manganese-induced neuronal apoptosis through activation of FOXO3a-Bim/PUMA axis. Sci Total Environ. 2019; 646:1047-55.

https://doi.org/10.1016/i.scitotenv.2018.07.363 PMID:30235590

39. Tseng PC, Hou SM, Chen RJ, Peng HW, Hsieh CF, Kuo $\mathrm{ML}$, Yen ML. Resveratrol promotes osteogenesis of human mesenchymal stem cells by upregulating RUNX2 gene expression via the SIRT1/FOXO3A axis. J Bone Miner Res. 2011; 26:2552-63.

https://doi.org/10.1002/jbmr.460 PMID:21713995

40. Sun W, Qiao W, Zhou B, Hu Z, Yan Q, Wu J, Wang R, Zhang $Q$, Miao D. Overexpression of Sirt1 in mesenchymal stem cells protects against bone loss in mice by FOXO3a deacetylation and oxidative stress inhibition. Metabolism. 2018; 88:61-71.

https://doi.org/10.1016/i.metabol.2018.06.006 PMID:30318050

41. Liang W, Li X, Li G, Hu L, Ding S, Kang J, Shen J, Li C, Asakawa T. Sirt1/Foxo Axis Plays a Crucial Role in the Mechanisms of Therapeutic Effects of Erzhi Pill in Ovariectomized Rats. Evid Based Complement Alternat Med. 2018; 2018:9210490.

https://doi.org/10.1155/2018/9210490 PMID:30224934

42. Zusso M, Lunardi V, Franceschini D, Pagetta A, Lo R, Stifani S, Frigo AC, Giusti P, Moro S. Ciprofloxacin and levofloxacin attenuate microglia inflammatory response via TLR4/NF-kB pathway. J Neuroinflammation. 2019; 16:148. https://doi.org/10.1186/s12974-019-1538-9 PMID:31319868 
43. Yan Z, Qi W, Zhan J, Lin Z, Lin J, Xue X, Pan X, Zhou Y. Activating Nrf2 signalling alleviates osteoarthritis development by inhibiting inflammasome activation. J Cell Mol Med. 2020; 24:13046-57. https://doi.org/10.1111/jcmm.15905

44. Afonina IS, Zhong Z, Karin M, Beyaert R. Limiting inflammation-the negative regulation of NF-KB and the NLRP3 inflammasome. Nat Immunol. 2017; 18:861-69. https://doi.org/10.1038/ni.3772 PMID:28722711

45. Fioravanti $A$, Tenti $S$, McAllister $M$, Chemaly $M$, Eakin A, McLaughlin J, Bjourson AJ, Frati E, McGilligan V,
Cheleschi S, Gibson DS. Exploring the Involvement of NLRP3 and IL-1 $\beta$ in Osteoarthritis of the Hand: Results from a Pilot Study. Mediators Inflamm. 2019; 2019:2363460.

https://doi.org/10.1155/2019/2363460

PMID: $\underline{30983879}$ 